\title{
1 Phosphatidylserine Receptors Enhance SARS-CoV-2 Infection: AXL as a Therapeutic
}

2 Target for COVID-19

3

4

5 Dana Bohan ${ }^{1 *}$, Hanora Van Ert ${ }^{1 *}$, Natalie Ruggio ${ }^{1}$, Kai J. Rogers ${ }^{1}$, Mohammad Badreddine ${ }^{1}$, 6 José A. Aguilar Briseño ${ }^{1}$, Roberth Anthony Rojas Chavez ${ }^{1}$, Boning Gao ${ }^{2}$, Tomasz Stokowy ${ }^{3}$,

7 Eleni Christakou ${ }^{3,4}$, David Micklem ${ }^{4}$, Gro Gausdal ${ }^{4}$, Hillel Haim ${ }^{1}$, John Minna ${ }^{2}$, James B. Lorens ${ }^{3}$

8 and Wendy Maury ${ }^{1 \dagger}$

$10{ }^{1}$ Department of Microbiology and Immunology, University of lowa, lowa City, IA

$11{ }^{2}$ Hamon Center for Therapeutic Oncology Research, University of Texas Southwestern Medical

12 Center, Dallas, TX

$13{ }^{3}$ Department of Biomedicine, University of Bergen, Bergen Norway.

$14{ }^{4}$ BerGenBio ASA, Bergen, Norway.

15 * These authors contributed equally to this work.

$16{ }^{\dagger}$ Corresponding author

18 Short Title: Phosphatidylserine Receptors Enhance SARS-CoV-2 Entry

19 Keywords: SARS-CoV-2, COVID19, phosphatidylserine receptor, apoptotic mimicry, viral entry,

20 AXL, bemcentinib, TIM-1, ACE2, TMPRSS2

21 Author summary word count: 191

22 Total character count (including spaces, excluding STAR Methods and supplemental

23 figure legends): 41,935 


\section{AUTHOR SUMMARY}

Phosphatidylserine (PS) receptors are PS binding proteins that mediate uptake of apoptotic bodies. Many enveloped viruses utilize this PS/PS receptor mechanism to adhere to and internalize into the endosomal compartment of cells and this is termed apoptotic mimicry. For viruses that have a mechanism(s) of endosomal escape, apoptotic mimicry is a productive route of virus entry. We evaluated if PS receptors serve as cell surface receptors for SARS-CoV-2 and found that the PS receptors, AXL, TIM-1 and TIM-4, facilitated virus infection when low concentrations of the SARS-CoV-2 cognate receptor, ACE2, was present. Consistent with the established mechanism of PS receptor utilization by other viruses, PS liposomes competed with SARS-CoV-2 for binding and entry. We demonstrated that this PS receptor enhances SARSCoV-2 binding to and infection of an array of human lung cell lines and is an under-appreciated but potentially important host factor facilitating SARS-CoV-2 entry.

\section{INTRODUCTION}

Severe Acute Respiratory Syndrome Coronavirus 2 (SARS-CoV-2) emerged in late 2019 and quickly spread around the world, resulting in the current public health pandemic. SARS-CoV-2 is a beta coronavirus of the sarbecovirus subgenus and is closely related to SARS-CoV, the agent responsible for an epidemic in 2003. SARS-CoV-2 is effectively transmitted between humans and has infected more than 178 million individuals and caused more than 3.88 million deaths worldwide as of June 23, 2021 (WHO). Fortunately, a herculean scientific effort has resulted in the development of SARS-CoV-2 vaccines which have been shown to be efficacious, potentially stemming the pandemic. Nonetheless, in combination with vaccines, continued development of efficacious antivirals is needed, as outbreaks continue in under-vaccinated regions and severe disease is not eradicated following vaccination. Towards this goal, a more comprehensive understanding of SARS-CoV-2 interactions with host cells will be required.

SARS-CoV-2 entry into cells is mediated by the viral spike glycoprotein (S) binding to

52 Angiotensin Converting Enzyme 2 (ACE2) (1-3). The S1 subunit of S binds to ACE2 while S2

53 mediates membrane fusion. Cleavage at the S1/S2 junction occurs during virus egress from

54 producer cells by the host protease furin which facilitates S1 binding to ACE2. A second site

55 termed S2' is also cleaved by the host proteases. Cleavage by TMPRSS2 at the cell surface 56 promotes fusion of the viral and host plasma membranes $(2,4)$. Alternatively, SARS-CoV-2

57 virions can be internalized via clathrin-mediated endocytosis after ACE2 binding, wherein host 
cathepsins (especially cathepsin L) proteolytically cleave S2' (3, 5-7). Adherence factors that enhance virion binding and increase infectivity have also been identified, namely neuropilin 1 and heparan sulfate $(8,9)$.

62 Binding and internalization of a variety of different enveloped viruses occurs through virion 63 associated phosphatidylserine (PS) binding to PS receptors. Members of the TIM family (TIM-1 64 and TIM-4) bind PS directly while another family of PS receptors, the TAM tyrosine kinase 65 receptor family (TYRO3, AXL and MERTK), bind PS indirectly through the adaptor proteins 66 Gas6 and Protein S. These PS receptor mediate binding and internalization of a wide range of 67 viruses, including filoviruses, alphaviruses, and flaviviruses (10-13). TIM-1, TIM-4, and AXL 68 appear to be the most efficacious at mediating viral entry given their prevalent use among enveloped viruses (14-16). Once virions are within the endosome, events that result in virion fusion with cellular membranes are virus specific, with filoviruses requiring viral glycoprotein processing followed by interactions with Niemann Pick C1 protein (NPC1) to initiate fusion, whereas flaviviruses rely on endosomal acidification driving glycoprotein conformational changes which mediate fusion $(17,18)$.

Given that PS receptors mediate entry of other enveloped viruses through interactions with viral membrane PS, we assessed the role of PS receptors on SARS-CoV-2 infection and the mechanism of interaction. We found that plasma membrane-expressed PS receptors by themselves do not result in productive SARS-CoV-2 infection; however, these receptors enhance infection when low levels of ACE2 are expressed. Our findings indicate that these receptors synergize with ACE2 to mediate SARS-CoV-2 entry through PS-dependent interactions. These data are in contrast to the conclusions drawn by another report stating that AXL interacts directly with the SARS-CoV-2 spike protein (19). Appreciation of this route of entry provides an additional pathway that could be therapeutically targeted to inhibit virus entry and subsequent infection.

\section{RESULTS}

\section{PS receptors enhance ACE2-dependent SARS-CoV-2 infection}

89 The ability of TIM and TAM family PS receptors to support SARS-CoV-2 infection were initially 90 examined in transfected HEK 293T cells. Wild-type HEK 293T cells do not express significant 91 amounts of ACE2 or PS receptors and are poorly permissive to SARS-CoV-2 infection $(3,20)$. 
92 Expression plasmids encoding ACE2 and/or the PS receptors, AXL or TIM-1, were transfected,

93 resulting in expression of these receptors on the surface of the transfected cells (S1A-B). Dual

94 transfection did not alter expression of ACE2 or PS receptors relative to single transfection. The

95 PS receptors AXL and TIM-1 by themselves did not facilitate infection of SARS-CoV-2 or VSV

96 pseudovirions bearing SARS-CoV-2 spike (VSV/Spike) (Fig. 1A, S1C). However, when low

97 levels of ACE2 (50 to $250 \mathrm{ng}$ of plasmid) were co-expressed with either AXL or TIM-1, the

98 combinations enhanced infection over that observed with ACE2 alone. TIM-1-enhanced ACE2-

99 dependent recombinant VSV/Spike (rVSV/Spike) (21) infection, and enhancement occurred

100 over a wider range of ACE2 concentrations than AXL-enhanced ACE2-dependent infection, with

101 AXL consistently enhancing infection when 250 ng of ACE2 plasmid was transfected (Fig. 1B-

102 C, S1C). The more limited ability of AXL to synergize was not due to limiting Gas6 in the media

103 as the addition of Gas6 to media did not enhance the synergy. At higher concentrations of

104 ACE2 plasmid, PS receptor enhancement of infectivity was reduced and, with transfection of 1

$105 \mu \mathrm{g}$ of ACE2 plasmid, no PS receptor enhancement was observed. Thus, only when ACE2 is

106 limiting on the cell surface do PS receptors facilitate infection. Consistent with a role for PS

107 receptors in SARS-CoV-2 entry, we observed enhanced virion attachment to cells when PS

108 receptors were expressed (Fig. 1D). In addition to viral load assessments, supernatants from

109 SARS-CoV-2 infected HEK 293T cultures were evaluated for production of infectious virions at

11048 hpi by TCID 50 assays in Vero E6 cells that express TMPRSS2. Low levels (50 ng) of ACE2

111 transfection increased virion production and this was enhanced by co-expression of TIM-1 (Fig.

112 1E). Consistent with the viral load findings, $A X L$ co-expression was not effective at enhancing

113 production of infectious virus when this low level of AXL was transfected. Other PS receptors,

114 TIM-4, TYRO3, and MerTK were examined for their ability of enhance infection. TIM-4

115 enhanced ACE2-dependent entry of VSV/Spike in a manner similar to TIM-1; however, TYRO3

116 and MerTK of the TAM family did not mediate increased entry, despite detectable levels of

117 plasma membrane expression after transfection (Fig. 1F; S1A-B; S1D). The synergy between

118 PS receptors and ACE2 was specific for SARS-CoV-2 as infection with VSV-luciferase

119 pseudovirions bearing Lassa virus GP was not affected by expression of these receptors (S1E).

120 These studies indicate that PS receptors synergize with low levels of ACE2 to enhance SARS-

121 CoV-2 infection. Further, these data provide evidence that VSV/Spike pseudovirions serve as a

122 useful BSL2 surrogate for SARS-CoV-2 entry events as others have shown (22-24).

123

124 PS receptors bind to virion associated PS, not the SARS-CoV-2 Spike protein 
125 We took several different approaches to examine the mechanism by which PS receptors

126 interact with SARS-CoV-2. In the context of other viral pathogens, PS receptors are known to

127 bind to virion membrane associated PS and mediate endosomal internalization of virions,

128 shuttling virus to cognate endosomal receptors. Liposomes composed of PS compete for virion

129 binding to PS receptors (25). To test this with SARS-CoV-2, increasing concentrations of PS or

130 phosphatidylcholine (PC) liposomes were evaluated for their ability to compete with virus for PS

131 binding sites in ACE2 + AXL or ACE2 + TIM-1 transfected HEK 293T cells. PS liposomes

132 effectively blocked VSV/Spike entry, whereas PC liposomes were significantly less effective

133 (Fig. 2A-B). We also assessed the activity of a TIM-1 mutant, ND115DN which has a disrupted

134 TIM-1 PS binding pocket, for its ability to facilitate VSV/Spike entry. This TIM-1 mutant did not

135 synergize with ACE2, indicating that the TIM-1 PS binding pocket is critical for this enhanced

136 activity (Fig. 2C) (12, 20).

138 Others have reported that the N-terminal domain of SARS-CoV-2 spike directly binds to AXL and is important for AXL-mediated entry of SARS-CoV-2 (19). To assess spike/AXL interactions, purified soluble full-length SARS-CoV-2 spike-Fc, spike receptor binding domain-

141 Fc (RBD) or spike N-terminal domain-Fc (NTD) was incubated with HEK 293T cells transiently

142 expressing AXL. Flow cytometry was used to detect spike proteins bound to AXL. Parallel ACE2

143 binding to soluble SARS-CoV-2 spike served as a positive control. As the spike NTD-Fc was

144 not expected to bind to ACE2, an ELISA confirmed the ability of a conformationally dependent

145 a-spike NTD monoclonal antibody to bind NTD-Fc, suggesting that NTD-Fc was in its native

146 conformation (S2B). The full-length spike-Fc and the RBD-Fc bound to ACE2, but no

147 interactions were detected between any of the purified spike proteins and AXL (Fig. 2D) despite

148 evidence of robust AXL surface expression on transfected HEK 293T cells (S2A) and the

149 equivalent levels of detection of the purified proteins via ELISAs (S2C). Biolayer interferometry

150 studies confirmed and extended our findings that recombinant AXL does not bind to purified

151 NTD, whereas interaction with the a-spike NTD monoclonal antibody was readily detected (Fig.

152 2E). Thus, using two complementary approaches, we were unable to demonstrate direct

153 interactions of AXL with spike. In total, our studies are consistent with PS receptors interacting

154 with SARS-CoV-2 virions through the well-established mechanism of virion-associated PS

155 binding to TIM-1 and AXL.

157 Redundant routes of virus entry: endosomal vs. plasma membrane mediated infection 
ACE2-dependent coronaviruses enter cells through two different routes: 1) An endosomal route of virus uptake that requires low $\mathrm{pH}$-dependent cathepsin $\mathrm{L}$ processing of spike, and 2) $\mathrm{A}$ plasma membrane route that is dependent upon TMPRSS2 cleavage of spike $(3,26)$. Others have reported that TMPRSS2-dependent entry is preferentially utilized by the virus when this serine protease is expressed (27). We examined the route of virus entry at play when ACE2, PS receptors and/or TMPRSS2 was expressed.

Initially, we assessed enhancement conferred by increasing amounts of TMPRSS2 plasmid on ACE2-dependent infection. As anticipated, we found that low levels (10 ng) of TMPRSS2 expressing plasmid enhanced VSV/Spike pseudovirion entry in HEK 293T cells co-transfected with $50 \mathrm{ng}$ of ACE2 expressing plasmid (Fig. 3A). However, at higher concentrations of TMPRSS2 plasmid, TMPRSS2 did not enhance infection, perhaps due to aberrant protease activity. Inverting these variables, when $10 \mathrm{ng}$ of TMPRSS2 plasmid was transfected in the presence of low concentrations of ACE2, TMPRSS2 enhanced virus infection (Fig. 3B). At high concentrations of ACE2, virus entry became TMPRSS2-independent in a manner similar to the effects of the PS receptors. Taken with Fig. 1B and C, these studies indicate that the PS receptors and TMPRSS2 can facilitate ACE2-dependent virus infection when ACE2 is limiting, but with increasing ACE2 concentrations the infections become independent of these entry factors. This may be related to effects of soluble ACE2 on entry (28).

To evaluate how PS receptors and/or TMPRSS2 expression would alter the route of ACE2dependent infection, HEK 293T cells were transfected PS receptors as before and infected with VSV/Spike in the presence or absence of the cysteine protease inhibitor, E-64, that blocks endosomal cathepsin activity. Non-toxic levels of E-64 were effective at blocking ACE2dependent infection (Fig. 3C, S3), indicating that virions were entering these cells in a cysteine protease-dependent manner, likely through the endosomal compartment. The enhancement of

184 virus entry conferred by the combination of PS receptors and ACE2 was also inhibited by E-64,

185 providing evidence that this is the route of virion uptake that is enhanced by PS receptors.

186 These findings are consistent with earlier reports that PS receptors mediate cargo

187 internalization into the endosomal compartment $(12,13,29)$. In cells that expressed ACE2 and

188 TMPRSS2, virus entry was no longer sensitive to E-64 as previously reported $(6,27)$.

189 VSV/Spike entry in the presence of TMPRSS2, PS receptors, and ACE2, was also insensitive to 190 E-64, suggesting that the TMPRSS2 expression and activity mediates entry at the plasma

191 membrane independent of PS receptor utilization. 


\section{Inhibition of endogenous AXL utilization blocks SARS-CoV-2 entry}

194 We next evaluated the ability of endogenously expressed PS receptors to enhance SARS-CoV-

1952 in ACE2 positive cells. Vero E6 cells that express ACE2, AXL, and TIM-1 (S4A) were initially

196 assessed (20). Notably, a large fraction of ACE2 protein is located intracellularly, suggesting a

197 rich reserve of ACE2 is inaccessible to extracellular virions (S4B). Initial studies using PS

198 liposomes confirmed that PS receptors are important for SARS-CoV-2 infection of these cells.

199 Competition studies in Vero E6 cells demonstrated that increasing doses of PS, but not PC,

200 inhibited VSV/Spike infection, similar to our findings in HEK 293T cells (Fig. 4A). PS liposomes

201 also significantly reduced SARS-CoV-2 binding to the surface of Vero E6 cells, implicating PS

202 receptors in attachment and subsequent entry of SARS-CoV-2 (Fig. 4B). These findings

203 reinforce the importance of either AXL, TIM-1, or both for SARS-CoV-2 entry.

To assess if AXL was important for infection of Vero E6 cells, the selective AXL kinase inhibitor, bemcentinib, was tested for its ability to block SARS-CoV-2 infection. In a dose dependent manner, bemcentinib profoundly inhibited SARS-CoV-2 virus load and blocked infection of the one-hit VSV/Spike pseudovirion (Fig. 4C, S4D). A time-of-addition study indicated that bemcentinib inhibition was most effective at early timepoints during SARS-CoV-2 infection, consistent with a role of AXL in virus entry (Fig. 4D). Bemcentinib toxicity was tested on human

211 lung epithelial cells and was nontoxic at the concentrations used (S4C). Consistent with an

212 important role for AXL in SARS-CoV-2 infection, RNAseq of infected Vero E6 demonstrated that

213 infection of these cells with a $\mathrm{MOI}$ of 0.01 resulted in $~ 80 \%$ of transcripts composed of viral 214 transcripts $18 \mathrm{hpi}$ (Fig. 4E). If these cells were treated with $1 \mu \mathrm{M}$ bemcentinib at the time of 215 infection, the fraction of viral transcripts dropped precipitously, decreasing to $\sim 10 \%$ of the total 216 reads.

218 To determine if TIM-1 contributed to SARS-CoV-2 infection of Vero E6 cells, the blocking anti219 human TIM-1 monoclonal antibody, ARD5, was evaluated for inhibition of recombinant VSV 220 (rVSV) bearing either Ebola GP (EBOV) or spike. While rVSV/EBOV GP was inhibited by ARD5 221 as previously reported $(12,30)$, it had no effect on rVSV/Spike infection (S4E). Thus, despite 222 robust expression of both PS receptors, AXL is preferentially utilized for SARS-CoV-2 infection 223 in these cells. While preferential PS receptor utilization has been reported for other pathogens 224 (20), our previous studies indicated that TIM-1 rather than AXL was preferentially used, in 225 contrast to our current observations with SARS-CoV-2. Host factors or virion attributes 
226 determining PS receptor preference are currently unexplored. A broad-spectrum TAM inhibitor,

227 BMS777607, modestly reduced virus infection in a dose dependent manner (Fig. 4F),

228 discounting the likelihood of off-target effects with bemcentinib.

As the bulk of ACE2 in Vero E6 cells is intracellular (S4B), surface expressed-AXL may be

231 facilitating SARS-CoV-2 uptake into the endosomal compartment where proteolytic processing 232 and ACE2 interactions mediate fusion of the viral envelope and cellular membranes. Previous

233 studies with the betacoronavirus responsible for the 2003-2004 outbreak, SARS-CoV,

234 demonstrated that ACE2 is found abundantly in the endosomal compartment, specifically co-

235 localizing with the early endosomal marker EEA1 (31). Further, at $3 \mathrm{hpi}$, SARS-CoV antigens

236 colocalize with vesicular ACE2 and that ACE2 formed notable vesicular puncta in the infected

237 cells (31). We utilized Stimulated Emission Depletion (STED) microcopy, leveraging the super

238 resolution capabilities of this platform to investigate ACE2 and AXL colocalization in uninfected

239 and infected Vero E6 cells. In uninfected cells, AXL and ACE2 were found on the plasma

240 membrane and intracellularly, but colocalize poorly (Fig. 4G and H). However, as shown in the

241 micrographs (white arrows) and the associated fluorescence intensity plot profiles (S4F yellow

242 lines highlight selected ROI), ACE2 and AXL demonstrate overlapping localization patterns

243 within cytoplasmic punctate structures. Pearson's correlation coefficients of infected and

244 uninfected cells calculated for AXL and ACE2 intensity demonstrate a significant increase in

245 colocalization values between this PS receptor and the cognate SARS-CoV-2 receptor in

246 infected cells, relative to mock counterparts. (Fig. 4H). These data support the possibility that

247 PS receptors enhance SARS-CoV-2 trafficking into these intracellular puncta where ACE2 is

248 abundant.

\section{AXL promotes SARS-CoV-2 infection in a range of lung cell lines}

251 In addition to ACE2, many lung cell lines express AXL $(19,32)$ (S5A). We evaluated these lines

252 for their ability to support SARS-CoV-2 infection and whether infection was sensitive to

253 bemcentinib inhibition. The panel of lung cells that were selected included A549

254 (adenocarcinoma) stably expressing ACE2, HCC1650 (NSCLC), HCC1944 (squamous), H1819

255 (adenocarcinoma), H2302 (adenocarcinoma) and Calu3 (adenocarcinoma).

257 These cells were inoculated with SARS-CoV-2 (MOI $=0.5$ unless otherwise noted) in the 258 presence or absence of a serial dilution of the AXL inhibitor, bemcentinib, or the cysteine 259 protease inhibitor, E-64. The cell lines A549ACE2, H1650, HCC1944, H1819, and HCC2302 
260 readily supported SARS-CoV-2 infection, and viral loads 24 hpi were decreased in a dose-

261 dependent manner, by bemcentinib and E64 (Fig. 5A-E). Infectious SARS-CoV-2 present in

262 HCC2302 cell supernatants at 24 and 48 hpi were also markedly decreased by bemcentinib

263 (Fig. 5F), demonstrating that bemcentinib treatment reduced production of new infectious virus

264 in a dose dependent manner. Further, at $1 \mu \mathrm{M}$ of bemcentinib, detectable production of any

265 infectious virus was delayed until 48 hours (L.O.D. = 5 TCID $50 / \mathrm{mL}$ ). In H1650 cells, the ability of

266 bemcentinib to inhibit recently emerged SARS-CoV-2 variants of concern (VOC), Alpha

267 (B.1.1.7) and Beta (B.1.351), was evaluated. While the Alpha VOC replicated poorly in these

268 cells, bemcentinib significantly inhibited virus replication of both variants, providing evidence the

269 efficacy of the AXL inhibitor is not influenced by SARS-CoV-2 adaptative changes (S5B).

SARS-CoV-2 infection of TMPRSS2 ${ }^{+}$Calu-3 cells (S6A) was not sensitive to bemcentinib or E-

27264 , again providing evidence that, in this cell line, the route of virus entry was dominated by the

273 TMPRSS2-dependent path, bypassing the use of PS receptors and the endosomal

274 compartment (Fig. 5G). These findings stand in contrast to SARS-CoV-2 infection of

275 TMPRSS2 ${ }^{+} \mathrm{H} 1650$ cells that were markedly bemcentinib and E-64 sensitive and were found to

276 be insensitive to camostat inhibition (Fig. 5B, S5C). The paradoxical finding that virus entry into

$277 \mathrm{H} 1650$ is sensitive to E64 and bemcentinib despite endogenous TMPRSS2 expression indicates

278 that TMPRSS2-dependent pathways are not always the dominating or default route of SARS-

279 CoV-2 entry and suggests that a more complex balance of events controls which pathway is

280 used. Neither the total amount of cell surface expressed ACE2 nor the intracellular versus

281 extracellular ACE2 ratio appears to determine the route of virus uptake (S5D).

RNA sequencing studies confirmed and extended our findings with bemcentinib in A549ACE2 cells. At $24 \mathrm{hpi}, 20 \%$ of the transcripts in A549ACE2 cells mapped to the viral genome. Infection in

285 the presence of $1 \mu \mathrm{M}$ bemcentinib significantly decreased the number of viral transcripts present

286 (Fig. 5H). Further analysis of potential qualitative changes in viral transcripts indicated that

287 transcript numbers across the genome were reduced, rather than a reduction of specific

288 subgenomic transcripts.

290 To directly evaluate the importance of AXL during infection of human lung cells, CRISPR-Cas9

291 technology was used to knock out (KO) AXL expression in H1650 and HCC2302 cells. H1650

$292 A X L^{\text {neg }}$, a biologically cloned $A X L$-null line, was evaluated along with bulk $A X L K O$ populations

293 of $\mathrm{H} 1650$ and $\mathrm{HCC} 2302$, denoted as $A X L^{\text {low }}$. The $A X L^{\text {neg }}$ clone, which expressed undetectable 
294 levels of AXL protein (Fig. 6A), supported dramatically lower SARS-CoV-2 virus loads at a

295 range of input MOls (Fig. 6B) and became unresponsive to bemcentinib (Fig. 6C),

296 demonstrating an important role for AXL in SARS-CoV-2 infection and indicating that

297 bemcentinib specifically targets AXL. Bulk populations of AXL low $\mathrm{H} 1650$ and HCC2302 also

298 supported reduced levels of SARS-CoV-2 infection and were poorly responsive to bemcentinib

299 (S6A-E). Taken together, the data presented here demonstrate that SARS-CoV-2 utilizes AXL

300 to enhance virion binding and entry in some lung cell lines, and that this mechanism can be

301 effectively disrupted in human lung cells by small molecule inhibitors and genetic ablation of

$302 \mathrm{AXL}$.

303

\section{AXL facilitates infection of other betacoronaviruses}

To assess the role of $A X L$ in the related betacoronavirus, mouse hepatitis virus (MHV strain A59), we investigated the ability of bemcentinib to inhibit infection in C57BL/6J mouse bone marrow derived macrophages (BMDMs) that express AXL (33-35). As MHV uses the mouse receptor CEACAM as its cognate receptor, this model allowed us to examine the role of $A X L$ with a coronavirus that is ACE2-independent (36). Bemcentinib added to BMDM cultures decreased virus load at 24 hours in a dose-dependent and an MOI-dependent manner (S7A-B). At higher MOls the effect of bemcentinib was diminished. Bemcentinib treatment also inhibited

312 MHV infection in peritoneal macrophages, an MHV permissive population phenotypically and 313 functionally distinct from BMDMs (S7C). These studies provide evidence that AXL facilitates 314 infection of multiple members of this enveloped virus family, independent of the cognate 315 receptor used by the virus.

\section{DISCUSSION}

319 Here, we demonstrate that PS receptors, AXL, TIM-1 and TIM-4, synergize with ACE2 to 320 mediate SARS-CoV-2 infection of HEK 293T cells when ACE2, the cognate receptor for the 321 virus, was expressed at low levels. PS receptors enhanced virion binding to cells in a PS-

322 dependent manner. At higher levels of ACE2 expression, a role for the PS receptors was no 323 longer observed. Similar findings were observed for TMPRSS2-facilitated, ACE2-dependent 324 infection, indicating that when ACE2 is expressed on the plasma membrane at high 325 concentrations these host proteins that assist SARS-CoV-2 entry are no longer required. 
A recent study reported that AXL mediates SARS-CoV-2 infection (19). That report suggested that AXL-mediated virus entry is independent of ACE2 and that AXL binds to the N-terminal domain of SARS-CoV-2 spike. Conclusions from our studies indicate that AXL and other PS receptors mediate enhancement of SARS-CoV-2 infection through interactions with virionassociated PS in an ACE2-dependent manner. We report several lines of evidence that are consistent with our contention that the PS receptors interact with virion-associated PS. First, PS liposomes abrogate binding and entry in a dose dependent manner. Second, interfering with PS/PS receptor complexes by mutating the TIM-1 PS binding pocket abrogates SARS-CoV-2 infection. These data are consistent with and support the well-established mechanism of PS receptor enhancement of enveloped virus infection $(11-13,37,38)$. Third, we directly tested whether AXL binds to purified Spike or NTD by flow cytometry and biolayer interferometry assays and were unable to detect any interaction. Finally, the ability of diverse PS receptors to enhance SARS-CoV-2 infection in HEK 293T cells lend support to the contention that these receptors interact with PS rather than viral spike on the surface of SARS-CoV-2 to mediate productive infection. Thus, we conclude that AXL does not interact with SARS-CoV-2 spike, nor does it mediate virus entry unilaterally. It should be noted that this is the first example of an enveloped virus that utilizes PS receptors in conjunction with low/moderate expression of a high affinity surface receptor.

A previous study concluded that the related coronavirus, SARS-CoV, was not productively internalized by PS receptors (11). However, with insights from our studies, an alternative explanation is that PS receptor enhancement of coronavirus entry is ACE2-dependent and sufficient quantities of ACE2 on the plasma membrane abrogate a role for PS receptors. Thus, PS receptors only facilitate SARS-CoV-2 entry under conditions where ACE2 is expressed at suboptimal levels, conditions that were likely not evaluated in the cited study but are found on ACE2 expressing cells such as Vero E6 cells and many patient derived lung cell lines. As ACE2

353 expression is low within the lung, such suboptimal conditions may be highly relevant during

354 SARS-CoV-2 infection $(39,40)$.

PS receptors have previously been shown to interact with PS on the surface of other enveloped 357 viruses such as filoviruses, alphaviruses and flaviviruses and mediate internalization into 358 endosomes $(11,41)$. However, PS receptor-dependent entry is a mechanism that is functionally 359 out-competed by high-affinity viral glycoprotein-host receptor interactions, such as that of Lassa 360 virus with $a$-dystroglycan $(16,20)$. In the case of Lassa virus entry PS receptors seem to serve 
361 as a backup entry mechanism, as these receptors only mediate virus internalization when the

362 high affinity surface receptor, a-dystroglycan, is not expressed.

Our studies provide evidence that PS receptors enhance SARS-CoV-2 binding to cells and mediate internalization into endosomes where cysteine proteases potentiate spike protein triggering and subsequent fusion events. Consistent with the utilization of this uptake pathway, the cysteine protease inhibitor E-64 effectively blocked ACE2 or ACE2/PS receptor entry in HEK 293T cells. This is also supported by our super resolution microscopy observations that $A X L$ and ACE2 colocalize during SARS-CoV-2 infection. However, in HEK 293T, the route of virus entry changes upon expression of TMPRSS2; virion entry is no longer sensitive to E-64. As others have reported, these findings are consistent with TMPRSS2-dependent entry dominating as the route of entry when TMPRSS2 is expressed $(24,42,43)$. We also investigated virus infection of a variety of lung lines that endogenously express TMPRSS2, AXL, and ACE2. While findings with Calu-3 cells were similar to that we observed in TMPRSS2-transfected HEK 293T cells, the other TMPRSS2 ${ }^{+}$lung lines, such as $\mathrm{H} 1650$ cells, remained sensitive to E-64 and the AXL signaling inhibitor, bemcentinib. Differences in the ability of TMPRSS2 expression to control the route of entry may be due to a fine balance of surface expression of the various receptors and should be explored in more detail.

Our data indicate that AXL serves as the most important PS receptor for SARS-CoV-2 infection of the TIM and TAM families and our studies with MHV implicated AXL in facilitating infection of additional coronaviruses. While AXL is abundant on lung epithelial cells, it is also present in many organs in the body, with the exclusion of neural tissues $(32,44)$. Thus, it is likely a role for AXL in SARS-CoV-2 infection is not only relevant to lung cell populations, but ACE2-expressing tissues suspected to be affected by COVID-19 such as the heart and kidneys $(45,46)$. We surmise that $A X L$-inhibiting therapeutics could function in tandem with other antivirals, protecting a number of organs from infection. Our data suggest that the efficacy of bemcentinib will persist as the virus evolves, inhibiting the VOCs Alpha and Beta effectively. By targeting host proteins such as AXL we dramatically reduce the potential selection for pathogen mutants that reduce or abolish antiviral activity. Given that currently utilized small molecule therapeutics such as remdesivir targeting viral proteins have shown limited efficacy and the benefits of antibody-rich

392 convalescent plasma is minimal, AXL inhibition by small molecule inhibitors such as

393 bemcentinib offers a novel route of attack to reduce SARS-CoV-2 entry and disease $(47,48)$. 
395 The preferential utilization of AXL rather than TIM-1 by SARS-CoV-2 in Vero E6 cells was

396 unexpected. In our previously studies, other enveloped viruses that utilize PS receptors, such as

397 filoviruses, use TIM-1 preferentially when both proteins are expressed (12, 20). Further, a recent

398 study identified that the TIM-1 IgV domain that contains the PS binding pocket serves as an

399 effective inhibitor of enveloped virus infection regardless of the PS receptor utilized for virus

400 uptake (49), consistent with the good affinity the TIM-1 PS binding pocket has for PS (50).

401 Nonetheless, when TIM-1 is not present in cells and AXL is the sole PS receptor expressed,

$402 \mathrm{AXL}$ is used by filoviruses and flaviviruses (11,13, 51-53). The subpar utilization of $A X L$

403 reported for other viruses may be due to the requirement for the adaptor protein, Gas6, to also

404 be present, expression patterns, or steric factors. As multiple proteins from a variety of different

405 PS receptors families can mediate uptake of apoptotic bodies, it is no surprise that PS receptor

406 interactions with viruses are likewise intricate. Further studies are needed to understand the

407 preferential use of AXL by SARS-CoV-2.

Bemcentinib, an orally bioavailable small molecule inhibitor of $A X L$, is currently in Phase II trials for non-small cell lung cancer (NSCLC) and a variety solid and hematological cancers

411 (ClinicalTrials.gov IDs: NCT03184571, NCT03184558). However, multiple screens have

412 identified bemcentinib as inhibitory to SARS-CoV-2 infection, bolstering this mechanism of entry

$413(54,55)$. Two phase 2 clinicals trial evaluating efficacy of bemcentinib in hospitalized COVID-19

414 patients are ongoing, with the first recently reporting short-term efficacy results

415 (https://clinicaltrials.gov/ct2/show/NCT04890509). In this exploratory, open-label study

416 bemcentinib was added to standard-of care (SoC) therapy to hospitalized patients (56). Though

417 the primary endpoints (time to improvement by 2 points on WHO ordinal scale or time to

418 discharge) showed a marginal benefit of bemcentinib treatment, there was evidence of

419 potentially meaningful clinical benefit in a key secondary endpoint which was avoidance of

420 deterioration. These interim data are promising and support further clinical investigations of this

421 AXL inhibitor for treating SARS-CoV-2.

422

423 The robust body of PS receptor research completed in the last decade and historical patterns of

424 zoonotic events (Ebola virus, Zika virus, coronaviruses) suggest that future emergent viral

425 pathogens are likely to utilize PS receptors to enhance entry and infection. The observations

426 reported here that PS receptors are utilized by a novel pandemic coronavirus support this

427 conclusion. This confluence of information provides insights into a new class of potential

428 therapeutics to stem future outbreaks, namely drugs aimed at inhibiting PS receptor activity. 
429 Further studies are required to determine the role of TIM and TAM use in vivo; however, our

430 studies demonstrate a role of PS receptors in SARS-CoV-2 infection in relevant cell populations

431 and further extend the importance of PS receptors in enveloped virus entry to coronaviruses.

432

\section{STAR METHODS}

434

435 RESOURCE AVAILABILITY

436 Lead contact:

437 Further information and request for resources and reagents should be directed to and will be

438 fulfilled by the lead contact Wendy J. Maury (wendy-maury@uiowa.edu).

Materials availability:

441 This study did not generate new reagents.

EXPERIMENTAL MODEL AND SUBJECT DETAILS

Ethics statement: This study was conducted in strict accordance with the Animal Welfare Act and the recommendations in the Guide for the Care and Use of Laboratory Animals of the

447 National Institutes of Health (University of lowa (UI) Institutional Assurance Number: \#A3021-

448 01). All animal procedures were approved by the UI Institutional Animal Care and Use 449 Committee (IACUC) which oversees the administration of the IACUC protocols and the study 450 was performed in accordance with the IACUC guidelines (Protocol \#8011280, Filovirus 451 glycoprotein/cellular protein interactions).

454 The mice (6-8 weeks old, female) used in these studies were obtained from the Jackson

455 Laboratory (C57BL6/J). The protocol (\#8011280) was approved by the Institutional Animal Care 456 and Use Committee at the University of lowa.

\section{Primary Cells and Immortal Cell Lines}

459 Bone marrow derived macrophages (BMDM) were isolated and cultured in RPMI-1640

460 supplemented with $10 \%$ Fetal Bovine Serum (FBS), $0.5 \mu \mathrm{g} / \mathrm{mL}$ of penicillin and streptomycin 461 (pen/strep) and $50 \mathrm{ng} / \mathrm{mL}$ murine M-CSF. Vero E6 cells (ATCC CRL-1586), Vero TMPRSS2, 462 Vero E6 and HEK 293T (ATCC CRL-11268) were cultured in Dulbecco's modified Eagle's 
463 medium (DMEM, GIBCO, Grand Island, NY) supplemented with 5-10\% FBS and 1\%

464 penicillin/streptomycin (GIBCO). Blasticidin $(5 \mu \mathrm{g} / \mathrm{mL})$ was added to media supporting Vero E6

465 TMPRSS2 cell growth. A549 ACE2 cells were generated by transduction of A549 (ATCC CCL-

466 185) with a codon-optimized ACE2 encoding lentivirus and selection with $10 \mu \mathrm{g} / \mathrm{mL}$ blasticidin.

467 Clonal populations were isolated and ACE2 expression verified by western blot. H1650,

468 HCC2302, HCC1944 and H1819 human lung lines were maintained in RPMI with 5-10\% FBS

469 and pen/strep. Cell lines were periodically tested for mycoplasma contamination (E-Myco kit,

470 Bulldog Bio, Portsmouth, $\mathrm{NH}$ ) and cured of contamination before use (Plasmocin, Invitrogen,

471 San Diego CA). Cell lines were authenticated periodically by ATCC (A549 and HEK 293T) or

472 the lab responsible for their generation (H1650, HCC2302, HCC1944, H1819, and Vero

473 TMPRSS2).

475 AXL knockout HCC2302 and H1650 were generated by transduction of parental cells with a

476 Cas9 encoding lentivirus (kind gift of Aloysius Klingelhutz, University of lowa) and selection in

$47710 \mu \mathrm{g} / \mathrm{mL}$ blasticidin for 10 days. Then cells were transduced with an Invitrogen LentiArray

478 CRISPR gRNA lentivirus targeting $A X L$, and subsequently selected in puromycin at $2 \mu \mathrm{g} / \mathrm{mL}$ for

4795 days. Cells were then lifted, stained for $A X L$, and sorted for $A X L^{\text {low }}$ cells at the University of

480 lowa Flow Cytometry Core on a FACSAria Fusion (Becton, Dickinson and Company, Franklin

481 Lakes, New Jersey). Bulk populations of $A X^{\text {low }}$ cells were used for experiments as noted, and

482 Clone \#4 was generated by sorting single $A X L^{\text {low }}$ cells into a 96 well plate. $A X L$ expression was

483 verified by flow cytometry on a FACSVerse (Becton, Dickinson and Company).

\section{Viruses}

486 Studies used the 2019n-CoV/USA-WA-1/2020 strain of SARS-CoV-2 (Accession number:

487 MT985325.1) which was propagated on Vero E6 cells. Briefly, Vero E6 cells were inoculated

488 with an MOI of 0.001 in DMEM supplemented with $2 \%$ FCS and pen/strep. Media was removed

489 and refreshed 24 hpi. When cells exhibited severe cytopathic effect, generally 72 hpi, cells were

490 freeze-thawed once, transferred to a conical tube, centrifuged at $1000 \mathrm{~g}$ for 10 minutes, and

491 supernatants were filtered through a $0.45 \mu \mathrm{m}$ filter. Virus was sequenced via Sanger

492 sequencing periodically for furin cleavage site mutations (none were detected) and only low

493 passage stocks were used. 
495 MHV (A59) stocks were generously provided by Dr. Stanley Perlman. Viral stocks were

496 generated on Vero E6 and the TCID 50 was determined on HeLa-mCECAM1 cells by

497 identification of cytopathic effect at 5 days.

498

499 Stocks of recombinant vesicular stomatitis virus that expressed SARS-CoV-2 Spike containing 500 the D614G mutation and nano-luciferase (rVSV/Spike) (kind gift of Dr. Melinda Brindley, Univ.

501 GA) were generated in either Vero E6 or Vero E6 TMPRSS2 cells. Cells were infected with a 502 low $\mathrm{MOI}$ ( 0.005) of virus and input was removed after $\sim 12 \mathrm{~h}$. Upon evidence of cytopathology,

503 supernatants were collected over a three-day period, filtered through a $0.45 \mu \mathrm{m}$ filter and frozen

504 at $-80^{\circ} \mathrm{C}$ until purified. Supernatants were thawed and centrifuged overnight at $7000 \times \mathrm{g}$ to

505 concentrate the virus. The virus pellet was resuspended in endotoxin-free PBS and layered over

506 a $20 \%$ sucrose/PBS cushion. Virus was pelleted through the cushion by centrifugation at

$50728,000 \mathrm{rpm}$ in a SW60Ti rotor (Beckman). The virus pellet was resuspended in PBS and the

508 TCID50 was determined on Vero E6 cells.

510 All viral titers were determined by a modified Spearman-Karber method as previously described

511 and reported as infectious units (IU)/mL (57).

513 METHOD DETAILS

514 Inhibitors

515 Bemcentinib (BGB324, R428) was provided by BerGenBio ASA (Bergen, Norway) and

516 dissolved in DMSO for in-vitro studies. BMS777607 (Millipore Sigma) was dissolved in DMSO.

517 E-64 (Millipore Sigma) was dissolved in DMSO.

\section{RNA isolation and qRT PCR}

520 Total RNA for PCR was extracted from cells or tissue using TRIzol (Invitrogen, Cat\# 15596018)

521 according to the manufacturer's protocol. Total isolated RNA $(1 \mu \mathrm{g})$ was reverse transcribed to

522 cDNA using the High-Capacity cDNA Reverse Transcription Kit (Applied Biosystems Cat\#

523 4368814). The resulting cDNA was used for amplification of selected genes by real-time

524 quantitative PCR using Power SYBR Green Master Mix (Applied Biosystems, Cat\# 4368708).

525 Data were collected on QuantStudio 3 and Ct values determined with the QuantStudio Data

526 Analysis software (Applied Biosystems). Averages from duplicate wells for each gene were

527 used to calculate relative abundance of transcripts relative to housekeeping genes (HPRT,

528 GAPDH, mouse cyclophilin) and presented as $2^{-\Delta \Delta C T}$. 


\section{VSV/Spike pseudovirus production}

531 The production of SARS-CoV-2-Spike vesicular stomatitis virus (VSV/Spike) pseudovirions has

532 been described previously (Hoffmann et al., 2020). Briefly, HEK 293T cells were seeded in 10

$533 \mathrm{~cm}$ tissue culture plates (CellTreat; Ref\# 229692). After 48 hours cells were transiently

534 transfected with a SARS-CoV-2-Spike pCG1 plasmid (a kind gift from Dr. Stefan Pohlman as

535 described in Hoffmann et al., 2020) using a standard polyethyleneimine (PEI) protocol. For this

536 transfection, one tube was prepared with $16 \mu \mathrm{g}$ of plasmid diluted in $1.5 \mathrm{~mL}$ of OPTI-Mem

537 (Gibco; Ref\# 31985-070). The second tube was prepared with PEl (1 mg/mL) diluted in $1.5 \mathrm{~mL}$

538 of OPTI-Mem at a concentration of $3 \mu \mathrm{l} / 1 \mu \mathrm{g}$ of DNA transfected. The tubes were then

539 combined, vortexed for 10-15 seconds and left to incubate at room temperature for 15 minutes.

540 The mixture was then added dropwise to the HEK 293T cells and returned to incubator

541 overnight. Twenty-four hours after transfection the cells were infected with a stock of replication

542 insufficient VSV virions expressing firefly luciferase that were pseudotyped with Lassa virus

543 glycoprotein on the viral membrane surface. The infection was incubated for $\sim 6$ hours at $37^{\circ} \mathrm{C}$,

544 was removed from the cells, and fresh media was added. Viral collection took place at 24- and

545 48-hours post-infection. Media supernatants were removed from the flasks, briefly spun down to

546 remove cellular debris ( $180 \mathrm{xg}$ for 1 minute) and filtered through a $0.45 \mu \mathrm{m}$ syringe-tip disk

547 PVDF filter (CellTreat; Ref\# 229745). Supernatants were then concentrated by a 16-hour

548 centrifugation step at $5380 \times \mathrm{g}$ at $4^{\circ} \mathrm{C}$. Pseudovirions were purified through a $20 \%$ sucrose

549 cushion via ultracentrifugation at $28,000 \mathrm{rpm}$ for two hours at $10^{\circ} \mathrm{C}$ in a Beckman Coulter

550 SW60Ti rotor. Pseudovirus was then resuspended in $1 \times \mathrm{PBS}$ and stored at $-80^{\circ} \mathrm{C}$. Pseudovirus

551 was titered using end point dilution on Vero E6 cells. All infections of cells using SARS-CoV-2-S

552 pseudotyped virions were conducted at a volume of virus that gave a relative light unit (RLU) of

553 roughly $100,000-200,000$ RLU.

554

555 HEK 293T transfections and plasmids

556 All transfections were performed in HEK 293T cells, with a total plasmid concentration of $2 \mu \mathrm{g}$.

557 Cells were seeded into 6-well tissue culture plate (Dot Scientific; Ref\# 667106) at a density of 5

$558 \times 10^{5}$ cells/well. Forty-eight hours after cell seeding, cells were transfected with CMV-driven

559 expression vectors of ACE2, TIM-1, TIM-4, AXL, TYRO3, MerTK and TMPRSS2 (see plasmid

560 details below) with a standard PEI transfection protocol. For this transfection one tube was

561 prepared with plasmid DNA and $150 \mathrm{mM} \mathrm{NaCl}$ at a concentration of $25 \mu \mathrm{l} / 1 \mu \mathrm{g}$ of DNA

562 transfected. A second tube was prepared with $150 \mathrm{mM} \mathrm{NaCl}$ at a concentration of $25 \mu \mathrm{l} / 1 \mu \mathrm{g}$ of 
563 DNA transfection along with PEl $(1 \mathrm{mg} / \mathrm{mL})$ at a concentration of $3 \mu \mathrm{l} / 1 \mu \mathrm{g}$ of DNA transfected.

564 Tubes were combined, vortexed vigorously for 10-15 seconds and incubated at room

565 temperature for 15 minutes. Mixtures were added dropwise to HEK 293T cells. For all

566 experiments using ACE2 50 ng of ACE2 plasmid were transfected, unless otherwise noted. For

567 PS receptors, 1000ng of plasmid was transfected unless otherwise noted. All transfections were

568 brought up to $2 \mu \mathrm{g}$ total transfected DNA with a PCD3.1 empty expression vector.

571 To detect cell surface expression of ACE and PS receptor on transfected HEK293Ts, WT

572 VeroE6s, and AXL knock down/out H1605 and HCC2302 clones, cells were lifted using 1x

573 Versene (GIBCO; Ref\# 15040066) at $37^{\circ} \mathrm{C}$ for $\sim 15$ minutes and placed in $5 \mathrm{~mL}$ polystyrene

574 round-bottom tubes (Falcon Ref\# 352052). Cells were washed once with FACS buffer (1x

575 Sterile PBS, 2\% FBS, 0.01\% sodium azide). Cells were incubated for 30 minutes on ice with

576 primary antibodies diluted in FACS buffer against ACE or PS receptors. Primary antibodies

577 were diluted to $0.75 \mu \mathrm{g} / \mathrm{mL}$ in FACS buffer prior to incubation. Specific primary antibodies used

578 as follows: goat anti-ACE2 (R\&D AF933), goat anti-AXL (R\&D154), goat anti-TIM-1 (R\&D 1750),

579 goat anti- Tyro3 (R\&D AF859), goat anti-TIM-4 (R\&D 2929), goat anti-MerTK (R\&D AF891),

580 rabbit anti-TMPRSS2 (Abcam ab92323). Cells were washed once with FACS buffer. Cells were

581 then incubated with secondary antibodies at a 1:1000 dilution in FACS buffer on ice for 30

582 minutes. Secondaries used were donkey anti-goat lgG $(\mathrm{H}+\mathrm{L})$ Alexa Fluor 647 (Jackson Immuno

583 Research; Ref\# 705-605-003) and donkey anti-rabbit IgG (H+L) Alexa Fluor 647 (Invitrogen;

584 A32733). Flowcytometry was performed on a Becton Dickinson FACS Calibur and analyzed by

585 Flow Jo.

586

587 To examine both the surface and intracellular expression of hACE2 on H1650, Calu3, 588 HCC1944, HCC2302, A549ACE2 and Vero E6 cells we performed the following protocol. Briefly, 589 cells were staining with Fixable Viability Dye eFluor 780 (eBiosciences), goat anti-human ACE2 590 (R\&D AF933) followed by secondary were donkey anti-goat lgG ( $\mathrm{H}+\mathrm{L})$ Alexa Fluor 647 591 (Jackson Immuno Research; Ref\# 705-605-003) To measure the intracellular expression of 592 hACE2, cells were surface stained with Fixable Viability Dye eFluor 780, fixed (PFA 4\%), 593 permeabilized (1X PBS + 0.5\%Tween20) and stained intracellularly using goat anti-human 594 ACE2 (R\&D AF933) followed by secondary donkey anti-goat labeled with AF647. Unstained 595 cells, cells plus viability dye and cells plus secondary antibody/viability dye were included as a 
control in every staining. Samples were measured on a FACSverse cytometer (BD Biosciences) and data were analyzed with Flowjo software (BD Biosciences).

\section{VSV/SARS-CoV-2-spike pseudovirion studies with inhibitors}

600 Following the transfection of HEK 293T cells, cells were incubated for 24 hours. At that time

601 cells were lifted with 0.25\% Trypsin (GIBCO; Ref\# 25200-056) and plated at a density of $2 \times 10^{4}$

602 cells/well on opaque, flat-bottomed, 96-well plates (Falcon; Ref\# 353296). Each transfection

603 was plated into at least 3 wells to create experimental replicates. Cells were incubated for an

604 additional 24 hours. At that time, cells were infected with VSV-luciferase/SARS-CoV-2 Spike.

605 Cells were incubated for an additional 24 hours. For experiments done with inhibitors, cells were

606 treated with the concentrations of inhibitors noted in the figure panel immediately prior to being

607 infected with pseudotyped virions. After $24 \mathrm{~h}$, virus-containing media was removed and replaced

608 with $35 \mu$ l of 1x Passive lysis buffer (Promega; Ref\# E194A). Plates underwent three freeze-

609 thaw cycles consisting of freezing on dry-ice for 15 minutes followed by thawing at $37^{\circ} \mathrm{C}$ for 15

610 minutes. We followed the protocol for measuring firefly luciferase as reported previously

611 (Johnson et al., 2017). For this method $100 \mu$ l of luciferin buffer (100 $\mu$ l of luciferin buffer (15

$612 \mathrm{mM} \mathrm{MgSO}_{4}, 15 \mathrm{mM} \mathrm{KPO}_{4}$ [pH7.8], $1 \mathrm{mM} \mathrm{ATP}$, and $1 \mathrm{mM}$ dithiothreitol) and $50 \mu \mathrm{l}$ of $1 \mathrm{mM} \mathrm{d}$ -

613 luciferin potassium salt (Syd Laboratories; Ref\# MB000102-R70170)) were added to each well

614 and luminescence was read via Synergy H1 Hybrid reader (BioTek Instruments). Relative

615 luminescence units were read out. Results analyzed by normalizing values to mock transfection

616 with no protease inhibitors.

\section{HEK 293T SARS-CoV-2 infection studies}

619 Following PEI transfection with plasmids as described in the previous section, cells were lifted

620 with $0.25 \%$ trypsin and plated into 48 -well plates at a density of $6 \times 10^{4}$ (Dot Scientific; Ref\#

621 667148). In our BSL3 facility, transfected HEK 293T cells were infected at a MOI = 0.5 with

622 SARS-CoV-2. Cells were incubated at $37^{\circ} \mathrm{C}$ or 24 hours and then treated with TRIzol RNA

623 isolation reagent and removed from the BSL3 facility. RNA extraction and cDNA generation

624 proceeded as described. qRT-PCR was conducted on the cDNA using SARS-CoV-2-Spike and

625 GAPDH primers. Data analyzed using the $\Delta \Delta \mathrm{Ct}$ method as described above.

626

\section{Virion binding assays}

628 For Vero E6 binding studies, cells were grown to confluence in 48 well plates. Media was

629 replaced with DMEM supplemented with $2 \%$ FBS and the indicated compounds. Cells were 
630 incubated at $10^{\circ} \mathrm{C}$ (preventing internalization and entry) until equilibrated and SARS-CoV-2 was

631 added at $\mathrm{MOI} 5$. Plates were returned to $10^{\circ} \mathrm{C}$ for 1 hour. Media was then removed and cells

632 were washed three times with cold DPBS (GIBCO, Cat\# 14190144), removing any unbound

633 virus. Then 0.05\% Trypsin-EDTA (GIBCO, Cat\# 25300054) was added to control wells for 5

634 minutes at $37^{\circ} \mathrm{C}$, and washed. After the final wash, all media was removed and replaced with

635 TRIzol (Invitrogen, Cat\# NCC1701D). RNA was isolated and analyzed as described. Binding

636 studies in HEK 293T cells were performed using rVSV-SARS-CoV-2 Spike virions (rVSV/Spike)

637 and binding was performed at room temperature in IMMULON 2HB flat bottom plates (Thermo

638 Scientific, Waltham, MA).

639

RNA sequencing and analysis

641 Following indicated treatments and infections that were performed in triplicate or quadruplicate,

642 Vero E6 and A549 ${ }^{A C E 2}$ cells in 6 well formats were homogenized using QIAShredder tubes and

643 total RNA isolated using the RNEasy kit with DNase treatment (Qiagen). High quality RNA

644 samples that were verified by a Bioanalyzer (Agilent) was quantified and used as input to

645 generate mRNA-seq libraries for the Illumina platform. Paired-end reads were performed at the

646 Paired-end sequencing reads were subject to alignment to suitable reference genomes: human

647 GRCh38 (GCA_000001405.15 - A549 cells), green monkey (Chlsab1/GCA_000409795.2 - Vero

648 E6 cells) and SARS-CoV-2 (MN985325 - both A549 and Vero E6 cells). Alignments to human

649 and monkey genomes were performed using hisat2 v2.0.5, while to viral genome using bowtie2

650 v2.2.9. Aligned reads were counted using featureCounts from subread package v1.5.2. Counted

651 reads were normalized in R, using DESeq2 v1.30.0 and subjected to statistical analysis. The

652 statistical analysis included computation of median based fold changes, Student t-test $p$ values

653 and false discovery rate (multiple testing correction).

655 Purified spike protein flow cytometry binding studies

656 The NTD-Fc and RBD-Fc constructs were kindly provided by Tom Gallagher. They contain the

657 Fc region of human IgG1 fused to the N-terminal domain of SARS-CoV-2 Spike (residues 1-

658 309) or the RBD-containing C-terminal domain of the S1 subunit (residues 310-529). We also

659 generated an Fc-Spike construct that contains the Fc region of human IgG1 fused to a

660 cleavage-negative form of the Spike ectodomain (subunits S1 and S2, corresponding to

661 positions 1-1274). To eliminate the polybasic furin cleavage site of Spike, we replaced the Arg-

662 Arg-Ala-Arg motif at positions 682-685 with Ser-Ser-Ala-Ser. All proteins were produced by

663 transient transfection of 293T cells using polyethylenimine. Proteins were harvested in 2935 
664 ProCDM and purified using Protein A beads. Eluted products were dialyzed against phosphate

665 buffered saline ( $\mathrm{pH}$ 7.4). All proteins were analyzed by SDS-PAGE and gels were silver stained

666 to verify the purity of the eluted product.

668 We measured the binding efficiency of anti-NTD antibody AM121 (Acro Biosystems) to the 669 Spike-based constructs using an enzyme-linked immunosorbent assay (ELISA), as previously 670 described $(58,59)$. For this purpose, the NTD-Fc, RBD-Fc or Spike-Fc suspended in PBS were 671 attached to 96-well protein-binding plates by incubation at isomolar concentrations (2, 1.37 and $6725 \mu \mathrm{g} / \mathrm{mL}$ of the probes, respectively). The next day, wells were washed once with buffer

673 containing $140 \mathrm{mM} \mathrm{NaCl}, 1.8 \mathrm{mM} \mathrm{CaCl}$, $1 \mathrm{mM} \mathrm{MgCl}$, $25 \mathrm{mM}$ Tris pH 7.5, $20 \mathrm{mg} / \mathrm{mL}$ BSA and $6741.1 \%$ low-fat milk. The anti-NTD antibody suspended in the same buffer was then added to the 675 wells at $0.5 \mu \mathrm{g} / \mathrm{mL}$. Binding of the anti-NTD antibody was detected using a goat anti-human 676 kappa light chain conjugated to horseradish peroxidase (HPR) (BioRad). To normalize for the 677 amount of the bound probes, we also quantified the amount of probe bound to the wells by 678 incubation with an HRP-conjugated goat anti-human antibody preparation. Binding of the HRP679 conjugated antibodies was measured by luminescence using SuperSignal West Pico enhanced 680 chemiluminescence reagents and a Synergy H1 microplate reader, as previously described 681 (60).

683 To determine binding of the above probes to AXL, we used flow cytometry. Briefly, HEK 293T 684 cells were seeded in 6-well plates (8.5E5 cells per well) and transfected the next day with $1.5 \mu \mathrm{g}$ 685 of empty vector or plasmids that express $A X L$ or the full-length form of human angiotensin 686 converting enzyme 2 (ACE2) using JetPrime transfection reagent (PolyPlus). Three days after 687 transfection, cells were detached using PBS supplemented with 7.5 mM EDTA and washed 688 once with washing buffer (PBS supplemented with $5 \%$ newborn calf serum). Cells were then 689 incubated with the NTD-Fc, RBD-Fc or Spike-Fc probes (at $5 \mu \mathrm{g} / \mathrm{mL}$ ) or anti-AXL antibody (at $6900.75 \mu \mathrm{g} / \mathrm{mL}$ ) in the same buffer for one hour on ice and were washed four times with washing 691 buffer. To detect binding of the Fc probes, we used a goat anti-human polyclonal antibody 692 preparation conjugated to Alexa 647. To detect binding of the anti-AXL antibody, we used a 693 goat anti-donkey polyclonal antibody preparation conjugated to Alexa 594. Secondary 694 antibodies were added at a 1:500 dilution and incubated with the cells on ice for one hour. Cells 695 were then washed and analyzed by flow cytometry. Staining was evaluated on a Becton 696 Dickinson FACS Calibur and analyzed by Flow Jo. 
699 Biolayer interferometry was performed on an OctetRed96 (Pall Forte-Bio, USA) using NiNTA-

700 (Forte-Bio 185101) or Streptavidin- (Forte-Bio 18-5019) coated Dip and Read biosensors for

701 immobilisation of His-tagged or biotin-tagged proteins respectively. All samples were diluted in

702 Kinetic Buffer (0.1\% BSA, 0.02\% Tween 20, 0.05\% Sodium azide in PBS). Biosensors were

703 equilibrated in Kinetic Buffer, and protein (His-tagged SARS-Cov-2 S1 protein NTD, Acro

704 Biosystems, S1D-C52H6; irrelevant control His-tagged $\beta 4$ integrin fibronectin type III domain,

705 generous gift from Petri Kursala laboratory; biotinylated tilvestamab anti-AXL antibody,

706 BerGenBio, Norway) at a concentration of $0.7 \mu \mathrm{M}$ was loaded for 10 minutes. A baseline was

707 taken for 2 minutes in Kinetic Buffer before testing association of the target binding protein for

70810 minutes ( $A X L$ extracellular domain fused to human IgG Fc region, BerGenBio $A S A, 2 \mu M$;

709 positive control Anti-SARS-Cov2 spike NTD Neutralizing antibody, Acro Biosystems, AM121,

$710 \quad 0.1 \mu \mathrm{M}$; IgG1 isotype control antibody, BioXcell, BE0297, 0.1 $\mu \mathrm{M})$. Results were analyzed in

711 Prism 9.1.1 for MacOS (GraphPad Software, USA) by aligning to the baseline values

712 immediately prior to association and subtracting signal from isotype control.

\section{STED sample preparation and image acquisition}

$71512 \mathrm{~mm}$ \#1.5 coverslips (Fisher Scientific; Ref\# 12-545-81P) were coated with 0.1\% bovine

716 Achilles' tendon collagen diluted in $1 \mathrm{x}$ sterile PBS. Collaged solution was incubated at $37^{\circ} \mathrm{C}$ for

7172 hours before being plated to $12 \mathrm{~mm}$ coverslips. Collagen was allowed to incubate on

718 coverslips 12 hours at $37^{\circ} \mathrm{C}$. Slips were then rinsed with $1 \times$ PBS and dried in $37^{\circ} \mathrm{C}$ incubator.

719 Slips were stored in sterile 1x PBS until use.

721 Vero E6 cells were plated onto collaged coated slips at 30,000 cells/slip. 24 hours after plating,

722 cells were either left uninfected, or infected with SARS-CoV02 (WA-1) at an MOI = 0.01 and

723 incubated for 24 hours. At that time cells were washed once with sterile $1 \times$ PBS and then fixed

724 with 4\% PFA solution (Electron Microscopy Sciences; Ref\# 15710) for 10 minutes at room

725 temperature. Following PFA fixation, cells were washed three times with 1x PBS and stored at $726 \quad 4^{\circ} \mathrm{C}$ until use.

728 For immunofluorescent staining, coverslips were incubated for 2 hours at RT with a blocking 729 buffer consisting of $1 \%$ Triton $X-100,0.5 \%$ sodium deoxycholate, $1 \%$ egg albumin and $0.01 \%$

730 sodium azide all suspended in 1x PBS. After 2-hour blockade, coverslips were incubated

731 overnight at $4^{\circ} \mathrm{C}$ with primary antibodies against hACE2 (goat anti-hACE2; R\&D AF933), and 
732 hAXL (rabbit anti-hAXL; Cell Signaling C89E7). Cells were washed three times with 1x PBS for

7335 minutes each. Samples with goat anti-hACE2 primary antibodies were first incubated with

734 donkey anti-goat IgG $(\mathrm{H}+\mathrm{L})$ Alexa Fluor 568 (Invitrogen; A-11057) for 1 hour at RT. Cells were

735 washed three times with 1x PBS for 5 minutes. Coverslips incubated with donkey anti-goat

736 Alexa Fluor 568 were then incubated with 5\% NGS (Sigma; G9023) for one hour at RT. Cells

737 were washed three times 1x PBS then incubated for one-hour room temperature with goat anti-

738 rabbit IgG $(\mathrm{H}+\mathrm{L})$ Alexa Fluor Plus 488 (Invitrogen; A32731TR). After washing three times for 5

739 minutes with 1x PBS cells were fixed to glass microscopy slides (Fisher Scientific; 12-550-15)

740 with 12ul of Prolong Glass mounting medium (Invitrogen; P36982). Coverslips cured for 48

741 hours before imaging.

Image acquisition was performed on the Leica SP8 3X STED confocal microscope equipped with an HC PL APO C32 100X/1.4 oil objective lens, and LAS X software (Leica Microsystems; version 3.5.5.19976) in the Central Microscopy Research Facility at the University of lowa. Excitation was performed using a white light laser set to $20 \%$ intensity. Depletion was performed with a $660 \mathrm{~nm}$ laser set at $7 \%$ intensity for Alexa Fluor 488, and $775 \mathrm{~nm}$ with $16.5 \%$ laser for Alexa Fluor 568 fluorophore. Depletion lasers were aligned using the auto STED alignment tool in the LAS X software. Laser strength and gain were adjusted to prevent pixel saturation. Images collected with two-line averages and 2 frame accumulations. Post image analyses include deconvolution using Huygens Professional Software and the deconvolution Wizard auto

752 functionality (Scientific Volume Imaging). Fluorescence intensity plot profiles were created using 753 ColorProfiler plug-in for ImageJ (Dimiter Prodanov). Pearson's correlation coefficients were

754 performed by using freehand ROI selection tool in ImageJ to outline individual cells and 755 performing colocalization calculations using the Colocalization Test plugin (Tony Collins et al.).

756 Depicted are the $R$ values for 20 cells across five separate fields imaged from one coverslip.

\section{QUANTIFICATION AND STATISTICAL ANALYSIS}

759 Statistical analysis was completed in GraphPad Prism v9.0.2 (GraphPad Software, San Diego, 760 CA). Quantification of flow cytometry data was completed in FlowJo v10.7.1 (Becton, Dickinson

761 \& Company, Ashland, OR). Statistical significance was defined as $p<0.05$, and denoted by a

762 single asterisk $\left(^{*}\right)$. Details regarding statistical tests used and exact values of $n$ can be found in

763 the corresponding figure legends. All data presented is representative of $n=3$ independent

764 experiments unless otherwise noted. 


\section{AUTHOR CONTRIBUTIONS}

See Supplemental Figure 8 for author contributions matrix.

\section{ACKNOWLEDGEMENTS}

770 This study was primarily supported by NIH/NIAID grant R01 Al134733 (WJM), NIH/NCI grants

771 P50 CA070907 (JM), U54 CA260560 (JM) and a contract from BerGenBio to WJM. DB was

772 supported by NIH grant T32AI007511 (Training in Mechanisms of Parasitism). HVE was

773 supported by T32 GM007337.

Kaitlyn Bohan graciously designed and created our graphical abstract image. The Cas9/BlastR lentiviral expression vector was generously provided by Aloysius Klingelhutz, PhD. The A549 ${ }^{\text {ACE2 }}$ cell line was kindly provided by Balaji Manicassamy, PhD. Paige Richards assisted isolation of the $\mathrm{H} 1650 \mathrm{AXL}^{\text {neg }}$ clone.

Declaration of Interests: G.G., D.M., and E.C. are employees of BerGenBio ASA, a company with financial interests in this field. Partial funding was provided by BerGenBio ASA. No other authors have competing interests to declare. The funders had no role in study design, data collection, data analysis, decision to publish, or preparation of the manuscript.

Data presented herein were obtained at the Genomics Division of the lowa Institute of Human

786 Genetics which is supported, in part, by the University of lowa Carver College of Medicine. The

787 authors would like to acknowledge vital assistance from Chantal Allamargot, PhD. Imaging was

788 performed using the Leica SP8 STED super resolution confocal available for use in the 789 microscopy core.

791 The data presented herein were obtained at the Flow Cytometry Facility, which is a Carver

792 College of Medicine / Holden Comprehensive Cancer Center core research facility at the

793 University of lowa. The facility is funded through user fees and the generous financial support of 794 the Carver College of Medicine, Holden Comprehensive Cancer Center, and lowa City

795 Veteran's Administration Medical Center.

797 FIGURE LEGENDS

798 Figure 1: PS receptors synergize with ACE2, enhancing SARS-CoV-2 infection of HEK

799 293T cells. A) Cells transfected with expression PS receptor plasmids, AXL or TIM-1, with or 
800

801

802

803

804

805

806

807

808

809

810

811

812

813

814

815

816

817

818

819

820

821

822

823

824

825

826

827

828

829

830

831

832

833

without $50 \mathrm{ng}$ of ACE2 and infected 48 hours later with SARS-CoV-2 (MOI = 0.5). Viral loads were determined 24 hours following infection. B-C) PS receptors, TIM-1 (B) and AXL (C), enhance rVSV-luciferase/Spike infection at low concentrations of ACE2 are transfected. D) Virus binding of cells transfected with PS receptor plasmids with or without $50 \mathrm{ng}$ of ACE2. rVSV/Spike was bound to transfected cells at 48 hpi and bound virus was measured via RTqPCR. E) Supernatants from SARS-CoV-2 infected (MOI=0.5) transfected HEK 293T cells were titered 48 hpi on Vero E6-TMPRSS2 and TCID ${ }_{50}$ calculated by Spearman-Karber equation. These studies were performed with transfection of $50 \mathrm{ng}$ of ACE2 plasmid. F) HEK 293T cells transfected with expression PS receptor plasmids, TYRO3 or TIM-4, with or without $50 \mathrm{ng}$ of ACE2 and infected 48 hours later with SARS-CoV-2 (MOI = 0.5). Viral loads were determined 24 hours following infection.

Data shown are pooled from at least 3 independent experiments (A, B, C, D, E, F). Data represented as means \pm SEM. Student's t-test $(\mathbf{A}, \mathbf{E})$ and multiple t-test $(\mathbf{B}, \mathbf{C})$, One-Way ANOVA with multiple comparisons (D\&F); asterisks represent $p<0.05$.

Figure 2: PS receptors interact with SARS-CoV-2 by binding to PS. A-B) PS liposomes interfere with rVSV-luciferase/Spike infection. HEK 293T cells transfected with TIM-1 plasmid and $50 \mathrm{ng}$ of ACE2 plasmid (A) or AXL plasmid and $50 \mathrm{ng}$ of ACE2 plasmid (B) were infected with rVSV-luciferase/Spike in the presence of increasing concentrations of PS or PC liposomes and assessed for luciferase activity at 24 hours following infection. C) HEK 293T cells were transfected with WT or PS binding pocket mutant TIM-1 plasmids with or without 50 ng of ACE2 expressing plasmid and infected 48 hours later with rVSV-luciferase/Spike pseudovirions.

Luminescence fold change were compared to mock transfected lysates that were set to a value of 1. D) AXL is unable to directly interact with purified, soluble SARS-CoV-2 spike/Fc. HEK 293T cells transfected with AXL or ACE2 were incubated with soluble spike protein (S1/S2)-Fc, S1 RBD-Fc or S1 NTD-Fc and subsequently incubated with an Alexa 647 secondary. Spike protein binding was detected by flow cytometry. E) AXL does not bind to the NTD of SARS-CoV-2 spike. Biolayer interferometry association curves show that immobilized AXL-Fc fails to interact with purified NTD of spike.

Data are pooled from at least 3 independent experiments $(\mathbf{A}, \mathbf{B})$ or are representative of at least 3 experiments (C, D, E). Data represented as means \pm SEM. Multiple t-test $(\mathbf{A}, \mathbf{B})$, One-way ANOVA with multiple comparisons $(\mathbf{C})$; asterisks represent $p<0.05$.

Figure 3: The route of SARS-CoV-2 entry is altered by TMPRSS2 expression. A) HEK 293T cells were transfected with ACE2 and TMPRSS2 as noted and infected at $48 \mathrm{~h}$ with VSVluciferase/Spike. At 24 hpi, luminescence activity was determined. Findings are shown relative 
834 to empty vector (Mock) transfected cells. Panel depicts one representative experiment. Students

835 t-tests. B) TMPRSS2 expression enhances rVSV-luciferase/Spike entry at low levels of ACE2

836 expression. HEK 293T cells were transfected as indicated and pseudovirion entry assessed by

837 measuring luminescence activity at $24 \mathrm{hpi}$. C) Transfected HEK 293T cells were transfected and

838 infected with VSV-luciferase/Spike at $48 \mathrm{~h}$ in the presence or absence of E-64 (300 $\mu \mathrm{M})$.

839 Luciferase activity was determined $24 \mathrm{hpi}$.

840 Data are pooled from at least 3 independent experiments $(\mathbf{B}, \mathbf{C})$ or are representative of at least

8413 experiments $(\mathbf{A})$. Data represented as means \pm SEM. Student's T-tests $(\mathbf{A})$ Multiple t-tests $(\mathbf{B})$,

842 Two-way ANOVA with row-wise multiple comparisons $(\mathbf{C})$; asterisks represent $\mathrm{p}<0.05$.

843 Figure 4: AXL has a prominent role in SARS-CoV-2 entry in Vero E6 cells. A) PS liposomes

844 interfere with SARS-CoV-2 pseudovirion entry. Vero E6 cells were treated with PS or PC

845 liposomes and incubated with VSV-GFP/Spike pseudovirions for 24 hours. Entry was detected

846 by GFP fluorescence. B) PS liposomes disrupt SARS-CoV-2 binding. Vero E6 cells were

847 incubated with SARS-CoV-2 $(\mathrm{MOI}=5)$ at $10^{\circ} \mathrm{C}$ for 1 hour, washed extensively, and viral load

848 assessed by RT-qPCR. C) AXL signaling inhibitor bemcentinib inhibits SARS-CoV-2 infection in

849 Vero E6 cells. Cells were treated with bemcentinib and infected with SARS-CoV-2 $(\mathrm{MOI}=0.01)$.

850 Viral loads were measured 24 hpi by RT-qPCR. D) Bemcentinib inhibition of SARS-CoV-2

851 infection is most efficacious at early time points during infection. Vero E6 cells were challenged

852 with SARS-CoV-2 $(\mathrm{MOI}=0.01)$ and treated with either the vehicle control or $1 \mu \mathrm{M}$ bemcentinib

853 at the indicated time. Viral loads were measured 24 hpi by RT-qPCR. E) Vero E6 cells were

854 treated with $1 \mu \mathrm{M}$ bemcentinib, infected with SARS-CoV-2 $(\mathrm{MOI}=0.01)$ and mRNA harvested

85518 hpi. mRNA was deep sequenced on an Illumina platform, and viral loads were calculated by

856 alignment to the SARS-CoV-2 genome. F) Broad spectrum TAM inhibitor BMS-777607 inhibits

857 SARS-CoV-2 infection in Vero E6 cells. Cells were treated with inhibitor at indicated

858 concentrations for 1 hour, challenged $(\mathrm{MOI}=0.01)$, and viral loads measured 24 hpi by RT-

859 qPCR G) STED micrographs showing staining for ACE2 (red) and AXL (green) and merged in

860 Vero E6 cells. Insets are enlarged images from regions highlighted by yellow rectangles. White

861 arrows indicate shared vesicular structures between the two channels. Yellow arrowheads

862 indicate objects that are only seen in one channel. Plot profiles are shown in S4F, representing

863 signal intensity along the yellow lines in the merged panels. H) Pearson's correlation coefficients

864 of ACE2 and AXL were calculated for $n=20$ mock and infected cells (ROI determined by cell

865 borders). 
866 Data are pooled from at least 3 independent experiments $(\mathbf{B}, \mathbf{D}, \mathbf{F})$ or are representative of at

867 least 3 experiments $(\mathbf{A}, \mathbf{C}, \mathbf{G}, \mathbf{H})$. Data are represented as means \pm SEM. Multiple t-tests $(\mathbf{A})$

868 student's t-test $(\mathbf{B}, \mathbf{C}, \mathbf{F}, \mathbf{H})$; asterisks represent $\mathrm{p}<0.05$.

869 Figure 5: AXL inhibition reduces SARS-CoV-2 infection in human lung cells. A-F) SARS-

870 CoV-2 infection is reduced by AXL inhibition in multiple human lung cell lines. In order:

$871 \mathrm{~A} 49^{\text {ACE2 }}, \mathrm{H} 1650, \mathrm{HCC} 1944, \mathrm{H} 1819, \mathrm{HCC} 2302$, Calu3 were treated with the indicated inhibitors

872 for 1 hour and challenged with SARS-CoV-2 $(\mathrm{MOI}=0.5)$ for 24 hours. Viral load was assessed

873 by RT-qPCR. G) HCC2302 cells were treated with bemcentinib at the indicated concentrations

874 for 1 hour and infected with SARS-CoV-2 (MOI =0.5). Input virus was removed 6 hpi and

875 supernatant was collected at 24 and $48 \mathrm{hpi}$ and titered by TCID $_{50}$ assays on Vero E6-TMPRSS2

876 cells. TCID $_{50} / \mathrm{mL}$ was calculated by the Spearmann-Karber method. H) A549 ${ }^{\text {ACE2 }}$ were treated

877 with bemcentinib as indicated, infected with SARS-CoV-2 (MOI = 0.5) and mRNA harvested 24

878 hpi. mRNA was sequenced, and viral loads calculated by alignment to the SARS-CoV-2

879 genome.

880 Data are pooled from at least 3 independent experiments $(\mathbf{F})$ or are representative of at least 3

881 experiments (A, B, C, D, E, G). Data represented as means \pm SEM. Student's t-test; asterisks

882 represent $\mathrm{p}<0.05$.

883 Figure 6: AXL knockout reduces viral loads and ablates inhibition by bemcentinib. A)

$884 \mathrm{H} 1650 \mathrm{AXL}$ knockout cells were generated by lentiviral transduction of Cas9 and gRNA

885 targeting AXL, followed by selection, enrichment, and biological cloning. Shown are flow

886 cytometry histograms depicting AXL surface staining (black) and secondary only background

887 (grey), demonstrating loss of AXL expression. B) H1650 AXL ${ }^{\text {neg }}$ and $\mathrm{H} 1650$ Cas9 (parental)

888 lines were challenged with SARS-CoV-2 at indicated MOls. At 24hpi, viral loads were assessed

889 by RT-qPCR. C) $\mathrm{H} 1650$ parental and $\mathrm{AXL}^{\text {neg }}$ lines were treated with indicated concentration of

890 bemcentinib for 1 hour and subsequently challenged with SARS-CoV-2 $(\mathrm{MOI}=0.5)$ and viral

891 loads determine by RT-qPCR 24 hpi.

892 Data are pooled from at least 3 independent experiments $(\mathbf{B}, \mathbf{C})$ or are representative of at least

8933 experiments $(\mathbf{A})$. Data represented as means \pm SEM. Multiple t-tests; asterisks represent $p<$

8940.05.

895

897 Supplemental Figure 1: PS receptors synergize with ACE2, enhancing SARS-CoV-2

898 infection of HEK 293T cells. A) Representative surface staining of receptors transfected into 
cells. B) Surface expression (MFI) of proteins in mock transfected (empty vector) and transfected HEK 293T at 48 hours after transfection. Background fluorescence is shown for secondary antibodies used in experiment (a-goat or rabbit secondaries). C) HEK 293T cells,

902 transfected PS receptors as noted with or without $250 \mathrm{ng}$ of ACE2 were transduced with rVSV-

903 luciferase/Spike. Transduction was assessed 24 hours later via luminescence. D) Expression of

904 MerTK did not affect rVSV-luciferase/Spike transduction in the presence of $250 \mathrm{ng}$ of

905 transfected ACE2 plasmid. E) Expression of ACE2, TIM-1 or AXL did not enhance infection of

906 VSV-luciferase/Lassa virus GP pseudovirions. HEK 293T cells were transfected with PS

907 receptor plasmids and 50 ng of ACE2 and infected 48 hours later. Panels C-E are shown as fold

908 change of luciferase activity in cell lysates relative to mock transfected lysates that were set to a 909 value of 1.

910 Data shown are pooled from at least three independent experiments (C, D, and E). Data

911 represented as means \pm SEM. One-Way ANOVA with multiple comparisons (C, D), Student's t-

912 test $(\mathbf{E})$; asterisks represent $\mathrm{p}<0.05$.

913 Supplemental Figure 2: PS receptors interact with SARS-CoV-2 by binding to PS. A) AXL

914 surface expression in transfected HEK 293T cells. B) Soluble purified S1/S2-Fc and NTD-Fc are

915 detected by an NTD monoclonal antibody by ELISA. C) All spike-Fc proteins bind and are

916 detected at equivalent levels of ELISA plates.

917 Supplemental Figure 3: The route of SARS-CoV-2 entry is altered by TMPRSS2

918 expression. ATPLite cytotoxicity assay in H1650 cells, 24 hours following treatment with E64.

919 Data are represented as means +/- SEM.

920 Supplemental Figure 4: AXL has a prominent role in SARS-CoV-2 entry in Vero E6 cells.

921 A) ACE2, AXL and TIM-1 surface expression MFI in Vero E6 cells, as assessed by flow

922 cytometry. Background fluorescence is shown for secondary antibodies used in experiment. B)

923 Cell surface versus intracellular ACE2 expression in VeroE6 cells. Indicated cells were lifted,

924 permeabilized as noted, and stained with anti-ACE2 unconjugated primary antibodies and Alexa

925647 secondaries. C) Bemcentinib toxicity 24 hours after treatment was measured by ATPlite

926 assay in H1650 cell line. D) VSV-GFP/Spike entry was measured by flow cytometry 24 hours

927 after challenge to Vero E6 cells treated with bemcentinib. E) Vero E6 were treated with ARD5

928 (TIM-1 blocking antibody) 1 hour before infection with rVSV /SARS-CoV-2 Spike or rVSV

929 /EBOV-GP (MOI = 0.01). Viral load was measured 24 hpi by RT-qPCR. F) Plot profiles of ACE2

930 and $A X L$ intensity are shown in from STED micrographs in Fig. 4G, representing signal intensity

931 along the yellow lines in the merged panels. 
932 Data in A, C, D and E are shown as means \pm SEM. Multiple t-tests were performed in $C$ and

933 Student's t-test was performed in D and E; asterisks represent $p<0.05$.

934 Supplemental Figure 5: AXL inhibition reduces SARS-CoV-2 infection in human lung

935 cells. A) Multiple SARS-CoV-2 permissive cell lines were stained for extracellular ACE2, AXL,

936 TIM-1, and TMPRSS2 protein, and expression was quantified by flow cytometry. Shown are

937 flow cytometry histograms depicting target surface staining (black line) and secondary only

938 background (grey shade) B) $\mathrm{H} 1650$ cells were treated with $1 \mu \mathrm{M}$ of bemcentinib and infected

939 with one of three different variants of SARS-CoV-2: WA-1; B.1.1.7 or B.1.351 (MOI=0.5 for all

940 variants). RNA was isolated at $24 \mathrm{hpi}$ and assessed for virus load. C) $\mathrm{H} 1650$ cells were infected

941 with SARS-CoV-2 $(\mathrm{MOI}=0.5)$ after treatment with the indicated concentration of camostat for 1

942 hour. Viral loads 24hpi were measured by qRT-PCR. D) Extracellular and intracellular staining

943 of ACE2 are shown in multiple cell lines. Presented as frequency positive cells.

944 Data represented as means \pm SEM. Student's t-test; asterisks represent $p<0.05$.

945 Supplemental Figure 6: AXL knockout reduces viral loads and ablates inhibition by

946 bemcentinib. A) H1650 and HCC2302 AXL knockout cells were generated by lentiviral

947 transduction of Cas9 and gRNA targeting AXL, followed by selection. These are designated

948 "Bulk $A X L$ " "Shown are flow cytometry histograms depicting AXL surface staining (black) and

949 secondary only background (grey), demonstrating complete loss of AXL expression in H1650

$950 \mathrm{AXL}^{\text {neg }}$. B) $\mathrm{H} 1650 \mathrm{AXL}$ low and H1650 Cas9 (parental) lines were challenged with SARS-CoV-2 at

951 indicated MOls for 24 hpi and viral loads assessed by RT-qPCR. C) H1650 parental and AXL low

952 lines were treated with indicated concentration of bemcentinib for 1 hour and subsequently

953 challenged with SARS-CoV-2 (MOI = 0.5) and viral loads determine by RT-qPCR 24 hpi. D-E)

954 As in B-C with HCC2302 cells.

955 Data are pooled from at least 3 independent experiments (B, C, D, E) or are representative of at

956 least 3 experiments $(\mathbf{A})$. Data represented as means \pm SEM. Multiple t-tests were performed;

957 asterisks represent $p<0.05$.

958 Supplemental Figure 7: A) Bone marrow derived macrophages from C57bl6/J mice were

959 treated as indicated for 1 hour, challenged with MHV (stain A59) at the indicated MOI. Viral

960 loads were assessed 24 hpi by RT-qPCR. B) BMDMs were treated with indicated

961 concentrations of bemcentinib for 1 hour, infected with $\mathrm{MHV}(\mathrm{MOI}=0.001)$ for 24 hours and

962 viral load assessed by RT-qPCR. C) As in B, MHV infection of peritoneal macrophages (MOI

$963=0.001)$ treated with indicated concentrations of bemcentinib. 
Data shown are representative of 3 independent experiments. Data represented as means \pm

SEM. Student's t-test; asterisks represent $p<0.05$.

\section{LITERATURE CITED}

968 1. Letko M, Marzi A, Munster V. Functional assessment of cell entry and receptor usage for SARSCoV-2 and other lineage B betacoronaviruses. Nat Microbiol. 2020;5(4):562-9.

2. Wrobel AG, Benton DJ, Xu P, Roustan C, Martin SR, Rosenthal PB, et al. Author Correction: SARS-CoV-2 and bat RaTG13 spike glycoprotein structures inform on virus evolution and furin-cleavage effects. Nat Struct Mol Biol. 2020;27(10):1001.

973 3. Hoffmann M, Kleine-Weber H, Schroeder S, Krüger N, Herrler T, Erichsen S, et al. SARS-CoV-2 Cell Entry Depends on ACE2 and TMPRSS2 and Is Blocked by a Clinically Proven Protease Inhibitor. Cell. 2020;181(2):271-80.e8.

4. Coutard B, Valle C, de Lamballerie X, Canard B, Seidah NG, Decroly E. The spike glycoprotein of the new coronavirus 2019-nCoV contains a furin-like cleavage site absent in CoV of the same clade. Antiviral Res. 2020;176:104742.

$9795 . \quad H o f f m a n n$ M, Kleine-Weber H, Pohlmann S. A Multibasic Cleavage Site in the Spike Protein of SARS-CoV-2 Is Essential for Infection of Human Lung Cells. Molecular Cell. 2020;78(4):779-+. 6. Matsuyama S, Nagata N, Shirato K, Kawase M, Takeda M, Taguchi F. Efficient activation of the severe acute respiratory syndrome coronavirus spike protein by the transmembrane protease TMPRSS2. J Virol. 2010;84(24):12658-64.

7. Glowacka I, Bertram S, Muller MA, Allen P, Soilleux E, Pfefferle S, et al. Evidence that TMPRSS2 Activates the Severe Acute Respiratory Syndrome Coronavirus Spike Protein for Membrane Fusion and Reduces Viral Control by the Humoral Immune Response. Journal of Virology. 2011;85(9):4122-34. 8. Cantuti-Castelvetri L, Ojha R, Pedro LD, Djannatian M, Franz J, Kuivanen S, et al. Neuropilin-1 facilitates SARS-CoV-2 cell entry and infectivity. Science. 2020.

9. Daly JL, Simonetti B, Klein K, Chen KE, Williamson MK, Anton-Plagaro C, et al. Neuropilin-1 is a host factor for SARS-CoV-2 infection. Science. 2020.

10. Kondratowicz AS, Lennemann NJ, Sinn PL, Davey RA, Hunt CL, Moller-Tank S, et al. T-cell immunoglobulin and mucin domain 1 (TIM-1) is a receptor for Zaire Ebolavirus and Lake Victoria Marburgvirus. Proceedings of the National Academy of Sciences of the United States of America. 2011;108(20):8426-31.

11. Jemielity S, Wang JJ, Chan YK, Ahmed AA, Li W, Monahan S, et al. TIM-family proteins promote infection of multiple enveloped viruses through virion-associated phosphatidylserine. PLoS Pathog. 2013;9(3):e1003232.

998 12. Moller-Tank S, Kondratowicz AS, Davey RA, Rennert PD, Maury W. Role of the phosphatidylserine receptor TIM-1 in enveloped-virus entry. J Virol. 2013;87(15):8327-41.

13. Meertens L, Carnec X, Lecoin MP, Ramdasi R, Guivel-Benhassine F, Lew E, et al. The TIM and TAM Families of Phosphatidylserine Receptors Mediate Dengue Virus Entry. Cell Host Microbe. 2012;12(4):544-57.

14. Rhein BA, Brouillette RB, Schaack GA, Chiorini JA, Maury W. Characterization of Human and Murine T-Cell Immunoglobulin Mucin Domain 4 (TIM-4) IgV Domain Residues Critical for Ebola Virus Entry. J Virol. 2016;90(13):6097-111.

15. Morizono K, Chen IS. Role of phosphatidylserine receptors in enveloped virus infection. J Virol. 2014;88(8):4275-90.

16. Fedeli C, Torriani G, Galan-Navarro C, Moraz ML, Moreno H, Gerold G, et al. AxI Can Serve as Entry Factor for Lassa Virus Depending on the Functional Glycosylation of Dystroglycan. Journal of Virology. 2018;92(5).

1011 17. Carette JE, Raaben M, Wong AC, Herbert AS, Obernosterer G, Mulherkar N, et al. Ebola virus entry requires the cholesterol transporter Niemann-Pick C1. Nature. 2011;477(7364):340-3.

18. Fritz R, Blazevic J, Taucher C, Pangerl K, Heinz FX, Stiasny K. The Unique Transmembrane Hairpin of Flavivirus Fusion Protein E Is Essential for Membrane Fusion. Journal of Virology.

$1015 \quad 2011 ; 85(9): 4377-85$. 
19. Wang S, Quu ZY, Hou YN, Deng XY, Xu W, Zheng TT, et al. AXL is a candidate receptor for SARS-CoV-2 that promotes infection of pulmonary and bronchial epithelial cells. Cell Research. $2021 ; 31(2): 126-40$.

20. Brouillette RB, Phillips EK, Patel R, Mahauad-Fernandez W, Moller-Tank S, Rogers KJ, et al. TIM-1 Mediates Dystroglycan-Independent Entry of Lassa Virus. J Virol. 2018;92(16).

21. Havranek KE, Jimenez AR, Acciani MD, Lay Mendoza MF, Reyes Ballista JM, Diaz DA, et al. SARS-CoV-2 Spike Alterations Enhance Pseudoparticle Titers and Replication-Competent VSV-SARSCoV-2 Virus. Viruses. 2020;12(12).

22. Case JB, Rothlauf PW, Chen RE, Kafai NM, Fox JM, Smith BK, et al. Replication-Competent Vesicular Stomatitis Virus Vaccine Vector Protects against SARS-CoV-2-Mediated Pathogenesis in Mice. Cell Host Microbe. 2020;28(3):465-74 e4.

23. Case JB, Rothlauf PW, Chen RE, Liu ZM, Zhao HY, Kim AS, et al. Neutralizing Antibody and Soluble ACE2 Inhibition of a Replication-Competent VSV-SARS-CoV-2 and a Clinical Isolate of SARSCoV-2. Cell Host \& Microbe. 2020;28(3):475-+.

24. Hoffmann M, Kleine-Weber H, Schroeder S, Kruger N, Herrler T, Erichsen S, et al. SARS-CoV-2 Cell Entry Depends on ACE2 and TMPRSS2 and Is Blocked by a Clinically Proven Protease Inhibitor. Cell. 2020;181(2):271-80 e8.

25. Kobayashi N, Karisola P, Pena-Cruz V, Dorfman DM, Jinushi M, Umetsu SE, et al. TIM-1 and TIM-4 glycoproteins bind phosphatidylserine and mediate uptake of apoptotic cells. Immunity. 2007;27(6):927-40.

26. Laporte M, Raeymaekers V, Van Berwaer R, Vandeput J, Marchand-Casas I, Thibaut HJ, et al. The SARS-CoV-2 and other human coronavirus spike proteins are fine-tuned towards temperature and proteases of the human airways. PLoS Pathog. 2021;17(4):e1009500.

1039

1040

27. Ou TL, Mou HH, Zhang LZ, Ojha A, Choe H, Farzan M. Hydroxychloroquine-mediated inhibition of SARS-CoV-2 entry is attenuated by TMPRSS2. Plos Pathogens. 2021;17(1).

28. Yeung ML, Teng JLL, Jia L, Zhang C, Huang C, Cai JP, et al. Soluble ACE2-mediated cell entry of SARS-CoV-2 via interaction with proteins related to the renin-angiotensin system. Cell. 2021;184(8):2212-28 e12.

29. Ravichandran KS. Find-me and eat-me signals in apoptotic cell clearance: progress and conundrums. J Exp Med. 2010;207(9):1807-17.

30. Kondratowicz AS, Lennemann NJ, Sinn PL, Davey RA, Hunt CL, Moller-Tank S, et al. T-cell immunoglobulin and mucin domain 1 (TIM-1) is a receptor for Zaire Ebolavirus and Lake Victoria Marburgvirus. Proc Natl Acad Sci U S A. 2011;108(20):8426-31.

31. Wang HL, Yang P, Liu KT, Guo F, Zhang YL, Zhang GY, et al. SARS coronavirus entry into host cells through a novel clathrin- and caveolae-independent endocytic pathway. Cell Research. 2008;18(2):290-301.

32. Morales A, Rojo Rello S, Cristobal H, Fiz-Lopez A, Arribas E, Mari M, et al. Growth ArrestSpecific Factor 6 (GAS6) Is Increased in COVID-19 Patients and Predicts Clinical Outcome. Biomedicines. 2021;9(4).

33. Segawa K, Yanagihashi Y, Yamada K, Suzuki C, Uchiyama Y, Nagata S. Phospholipid flippases enable precursor B cells to flee engulfment by macrophages. Proc Natl Acad Sci U S A. 2018;115(48):12212-7.

1058

1059

1060

1061

1062

1063

1064

1065

1066

1067

1068

1069

34. Subramanian M, Proto JD, Matsushima GK, Tabas I. Deficiency of AXL in Bone Marrow-Derived Cells Does Not Affect Advanced Atherosclerotic Lesion Progression. Sci Rep. 2016;6:39111.

35. Seitz HM, Camenisch TD, Lemke G, Earp HS, Matsushima GK. Macrophages and dendritic cells use different Axl/Mertk/Tyro3 receptors in clearance of apoptotic cells. Journal of Immunology. 2007;178(9):5635-42.

36. Dveksler GS, Pensiero MN, Cardellichio CB, Williams RK, Jiang GS, Holmes KV, et al. Cloning of the mouse hepatitis virus (MHV) receptor: expression in human and hamster cell lines confers susceptibility to MHV. J Virol. 1991;65(12):6881-91.

37. Rhein BA, Maury WJ. Ebola Virus Entry into Host Cells: Identifying Therapeutic Strategies. Current Clinical Microbiology Reports. 2015;2(3):115-24.

38. Moller-Tank S, Albritton LM, Rennert PD, Maury W. Characterizing Functional Domains for TIMMediated Enveloped Virus Entry. J Virol. 2014;88(12):6702-13. 
39. Hou YJ, Okuda K, Edwards CE, Martinez DR, Asakura T, Dinnon KH, 3rd, et al. SARS-CoV-2

1071

1072

1073

1074

1075

1076

1077

1078

1079

1080

1081

1082

1083

1084

1085

1086

1087

1088

1089

1090

1091

1092

1093

1094

1095

1096

1097

1098

1099

1100

1101

1102

1103

1104

1105

1106

1107

1108

1109

1110

1111

1112

1113

1114

1115

1116

1117

1118

1119

1120

1121

1122

1123

1124

Reverse Genetics Reveals a Variable Infection Gradient in the Respiratory Tract. Cell. 2020;182(2):42946 e14.

40. Sungnak W, Huang N, Becavin C, Berg M, Queen R, Litvinukova M, et al. SARS-CoV-2 entry factors are highly expressed in nasal epithelial cells together with innate immune genes. Nat Med. 2020;26(5):681-7.

41. Moller-Tank S, Maury W. Phosphatidylserine receptors: enhancers of enveloped virus entry and infection. Virology. 2014;468-470:565-80.

42. Shang J, Wan Y, Luo C, Ye G, Geng Q, Auerbach A, et al. Cell entry mechanisms of SARS-CoV2. Proc Natl Acad Sci U S A. 2020;117(21):11727-34.

43. Shirato K, Kawase M, Matsuyama S. Wild-type human coronaviruses prefer cell-surface TMPRSS2 to endosomal cathepsins for cell entry. Virology. 2018;517:9-15.

44. Uhlen M, Fagerberg L, Hallstrom BM, Lindskog C, Oksvold P, Mardinoglu A, et al. Proteomics. Tissue-based map of the human proteome. Science. 2015;347(6220):1260419.

45. Liu H, Gai S, Wang X, Zeng J, Sun C, Zhao Y, et al. Single-cell analysis of SARS-CoV-2 receptor ACE2 and spike protein priming expression of proteases in the human heart. Cardiovasc Res. 2020;116(10):1733-41.

46. Puelles VG, Lutgehetmann M, Lindenmeyer MT, Sperhake JP, Wong MN, Allweiss L, et al. Multiorgan and Renal Tropism of SARS-CoV-2. N Engl J Med. 2020;383(6):590-2.

47. Simonovich VA, Burgos Pratx LD, Scibona P, Beruto MV, Vallone MG, Vazquez C, et al. A Randomized Trial of Convalescent Plasma in Covid-19 Severe Pneumonia. N Engl J Med. 2021;384(7):619-29.

48. Beigel JH, Tomashek KM, Dodd LE, Mehta AK, Zingman BS, Kalil AC, et al. Remdesivir for the Treatment of Covid-19 - Final Report. N Engl J Med. 2020;383(19):1813-26.

49. Song D-H, Garcia G, Situ K, Chua BA, Hong MLO, Do EA, et al. Development of a blocker of the universal phosphatidylserine- and phosphatidylethanolamine-dependent viral entry pathways. Virology. 2021;560:17-33.

50. Dragovich MA, Fortoul N, Jagota A, Zhang W, Schutt K, Xu Y, et al. Biomechanical characterization of TIM protein-mediated Ebola virus-host cell adhesion. Scientific reports. 2019;9(1):267. 51. Brindley MA, Hunt CL, Kondratowicz AS, Bowman J, Sinn PL, McCray PB, Jr., et al. Tyrosine kinase receptor $\mathrm{Axl}$ enhances entry of Zaire ebolavirus without direct interactions with the viral glycoprotein. Virology. 2011;415(2):83-94.

52. Zhang L, Richard AS, Jackson CB, Ojha A, Choe H. Phosphatidylethanolamine and Phosphatidylserine Synergize To Enhance GAS6/AXL-Mediated Virus Infection and Efferocytosis. J Virol. 2020;95(2).

53. Richard AS, Shim BS, Kwon YC, Zhang R, Otsuka Y, Schmitt K, et al. AXL-dependent infection of human fetal endothelial cells distinguishes Zika virus from other pathogenic flaviviruses. Proc Natl Acad Sci U S A. 2017;114(8):2024-9.

54. Dittmar M, Lee JS, Whig K, Segrist E, Li M, Kamalia B, et al. Drug repurposing screens reveal cell-type-specific entry pathways and FDA-approved drugs active against SARS-Cov-2. Cell Rep. 2021;35(1):108959.

55. Bouhaddou M, Memon D, Meyer B, White KM, Rezelj VV, Correa Marrero M, et al. The Global Phosphorylation Landscape of SARS-CoV-2 Infection. Cell. 2020;182(3):685-712 e19.

56. Godfrey R. BERGENBIO ANNOUNCES UPDATE FROM INVESTIGATIONAL PHASE II TRIALS ASSESSING BEMCENTINIB IN HOSPITALISED COVID-19 PATIENTS bergenbio.com2021 [updated April 19, 2021; cited 2021. Available from: https://www.bergenbio.com/bergenbio-announces-updatefrom-investigational-phase-ii-trials-assessing-bemcentinib-in-hospitalised-covid-19-patients/.

57. Ramakrishnan MA. Determination of $50 \%$ endpoint titer using a simple formula. World $\mathrm{J}$ Virol. 2016;5(2):85-6.

58. Johnson J, Flores MG, Rosa J, Han CZ, Salvi AM, DeMali KA, et al. The High Content of Fructose in Human Semen Competitively Inhibits Broad and Potent Antivirals That Target High-Mannose Glycans. Journal of Virology. 2020;94(9).

59. DeLeon O, Hodis H, O'Malley Y, Johnson J, Salimi H, Zhai Y, et al. Accurate predictions of population-level changes in sequence and structural properties of HIV-1 Env using a volatility-controlled diffusion model. PLoS Biol. 2017;15(4):e2001549. 
bioRxiv preprint doi: https://doi.org/10.1101/2021.06.15.448419; this version posted June 24, 2021. The copyright holder for this preprint (which was not certified by peer review) is the author/funder, who has granted bioRxiv a license to display the preprint in perpetuity. It is made available under aCC-BY 4.0 International license.

1125 60. Salimi H, Johnson J, Flores MG, Zhang MS, O'Malley Y, Houtman JC, et al. The lipid membrane

1126 of HIV-1 stabilizes the viral envelope glycoproteins and modulates their sensitivity to antibody

1127 neutralization. Journal of Biological Chemistry. 2020;295(2):348-62. 
Figure 1: PS receptors synergize with ACE2, enhancing SARS-CoV-2 infection.

A

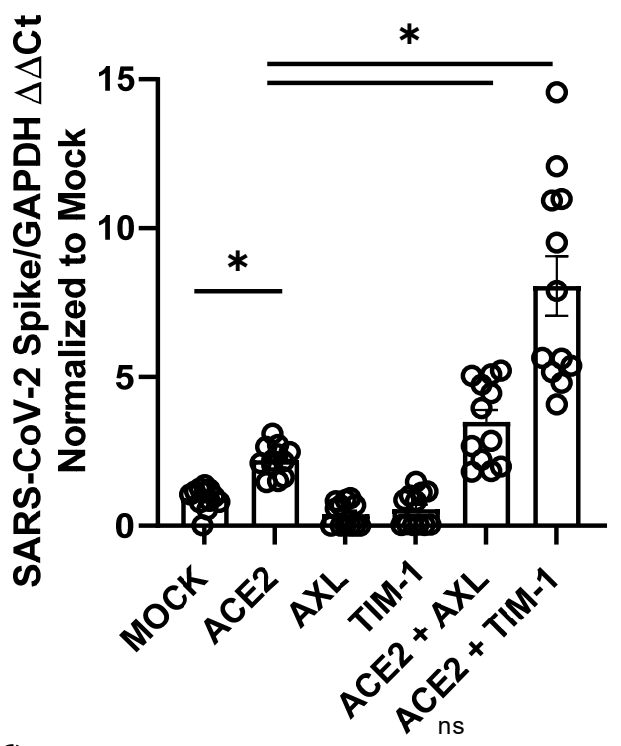

C

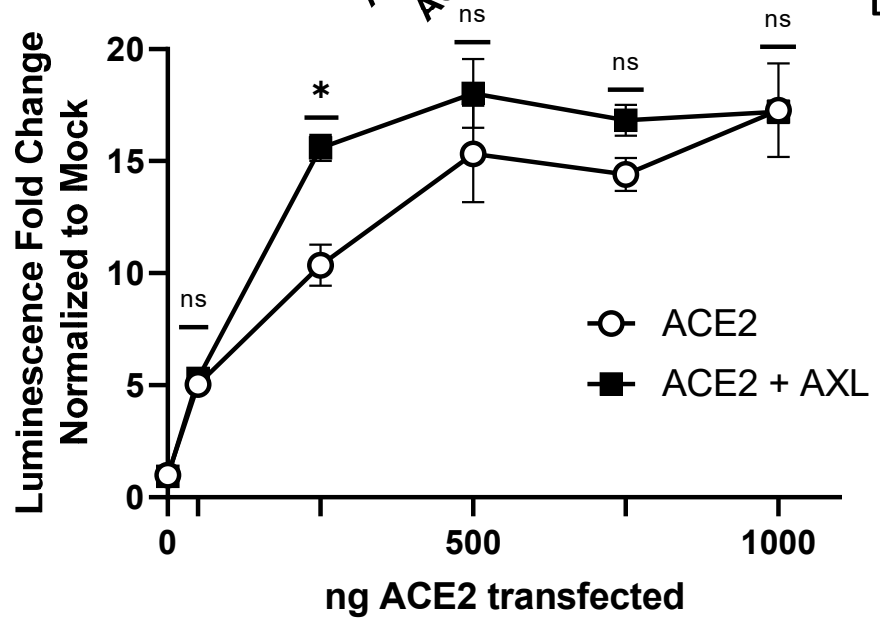

$\mathrm{E}$

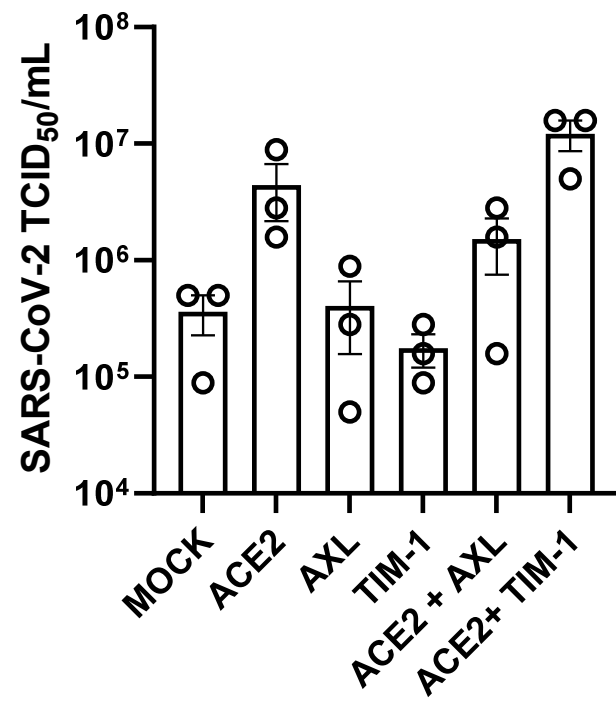

B

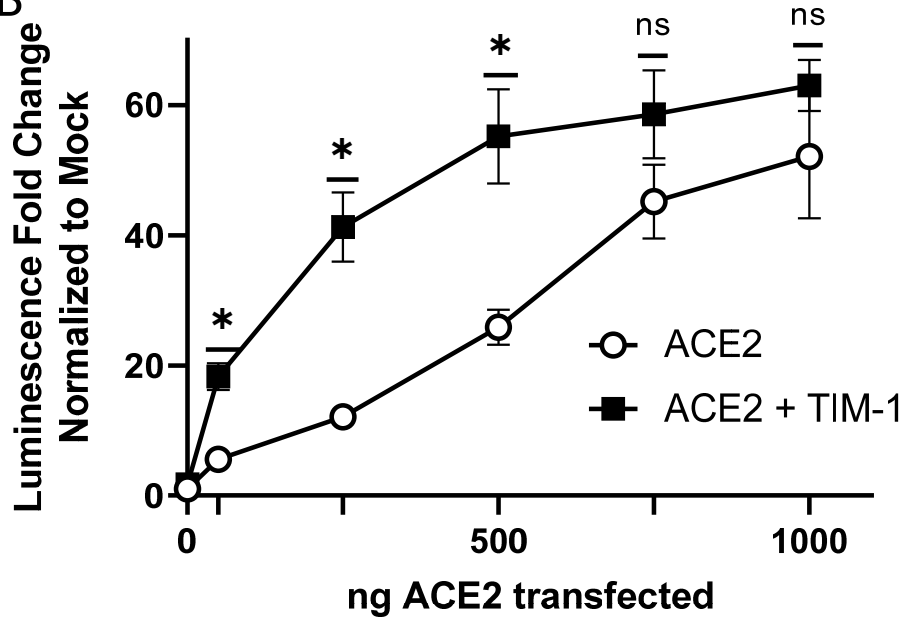

D

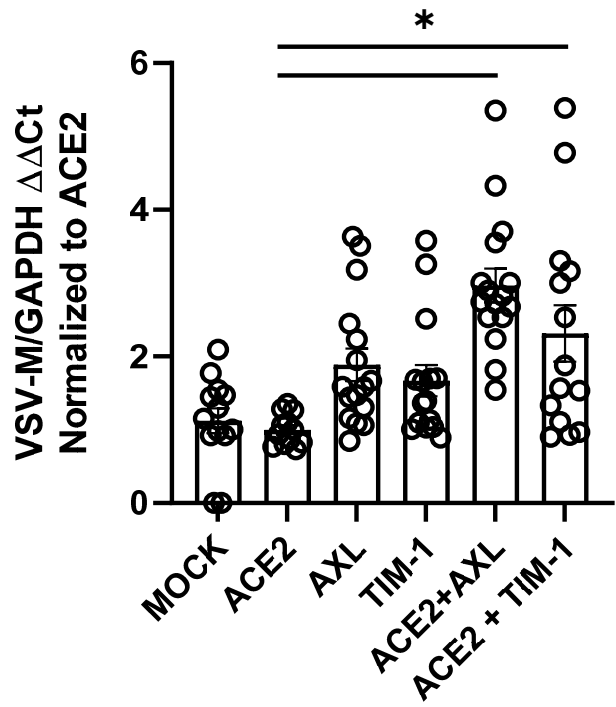

F

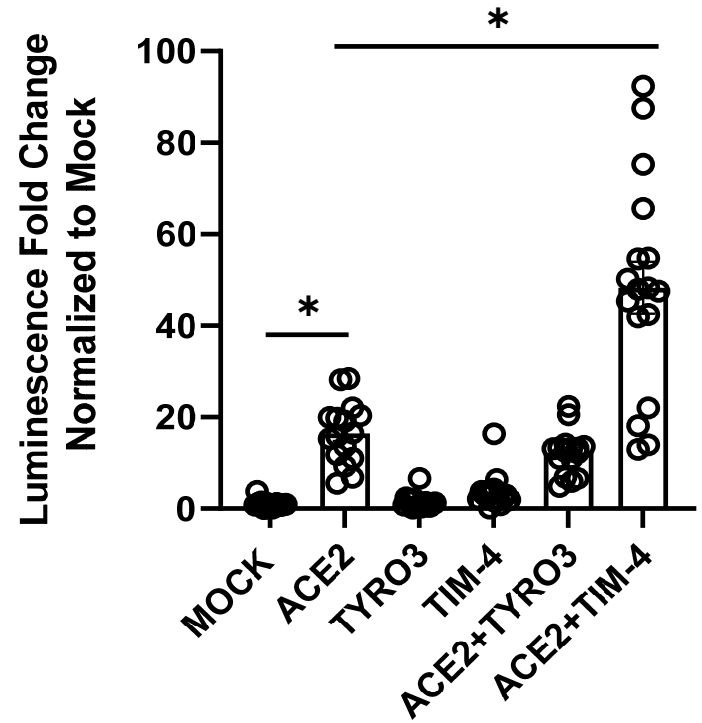


Figure 1: PS receptors synergize with ACE2, enhancing SARS-CoV-2 infection of HEK 293T cells. A) Cells transfected with expression PS receptor plasmids, AXL or TIM-1, with or without 50 ng of ACE2 and infected 48 hours later with SARS-CoV-2 (MOI =0.5). Viral loads were determined 24 hours following infection. B-C) PS receptors, TIM-1 (B) and AXL (C), enhance rVSVluciferase/Spike infection at low concentrations of ACE2 are transfected. D) Virus binding of cells transfected with PS receptor plasmids with or without $50 \mathrm{ng}$ of ACE2. rVSV/Spike was bound to transfected cells at $48 \mathrm{hpi}$ and bound virus was measured via RT-qPCR. E) Supernatants from SARS-CoV-2 infected $(\mathrm{MOI}=0.5)$ transfected HEK 293T cells were titered 48 hpi on Vero E6-TMPRSS2 and TCID $_{50}$ calculated by Spearman-Karber equation. These studies were performed with transfection of 50 ng of ACE2 plasmid. F) HEK 293T cells transfected with expression PS receptor plasmids, TYRO3 or TIM-4, with or without 50 ng of ACE2 and infected 48 hours later with SARS-CoV-2 (MOI = 0.5). Viral loads were determined 24 hours following infection.

Data shown are pooled from at least 3 independent experiments (A, B, C, D, E, F). Data represented as means \pm SEM. Student's t-test $(A, E)$ and multiple t-test $(B, C)$, One-Way ANOVA with multiple comparisons (D\&F); asterisks represent $p<0.05$. 
Figure 2: PS receptors interact with SARS-CoV-2 by binding to PS.

A

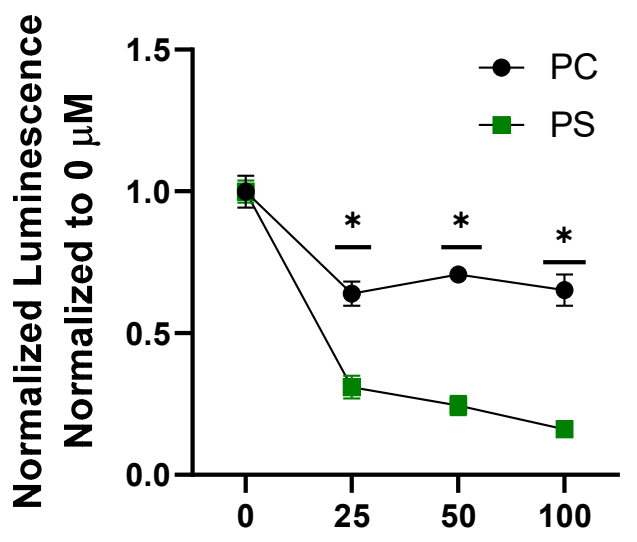

[Liposomes] $\mu \mathrm{M}$
B

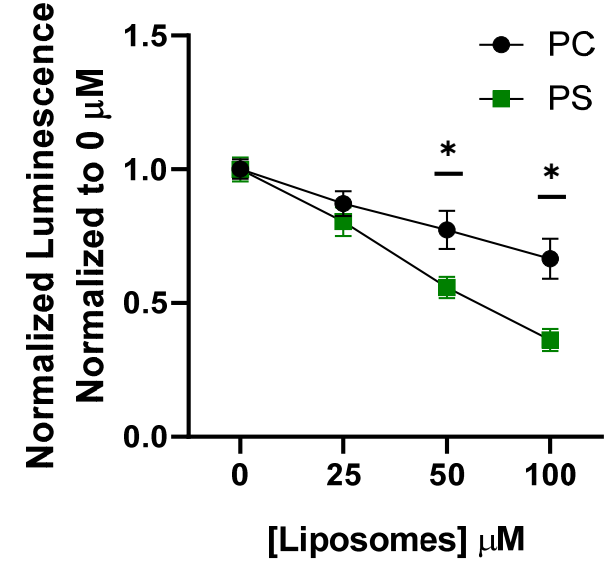

$\mathrm{E}$

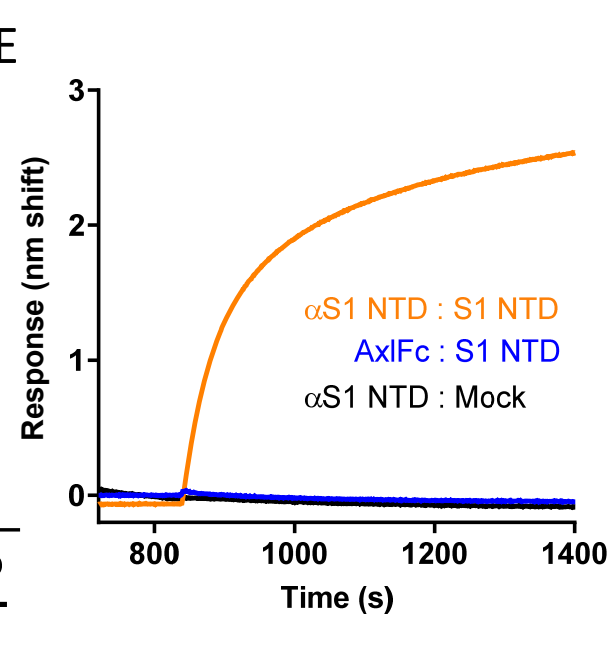

[Liposomes] $\mu \mathrm{M}$
C

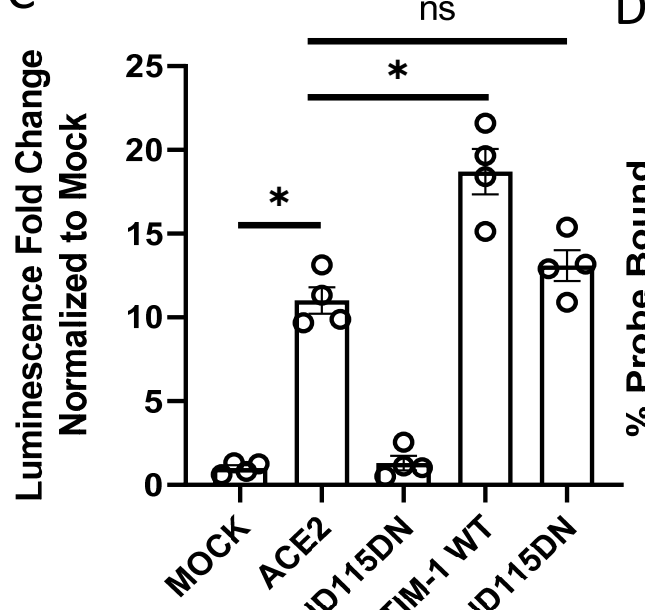

D

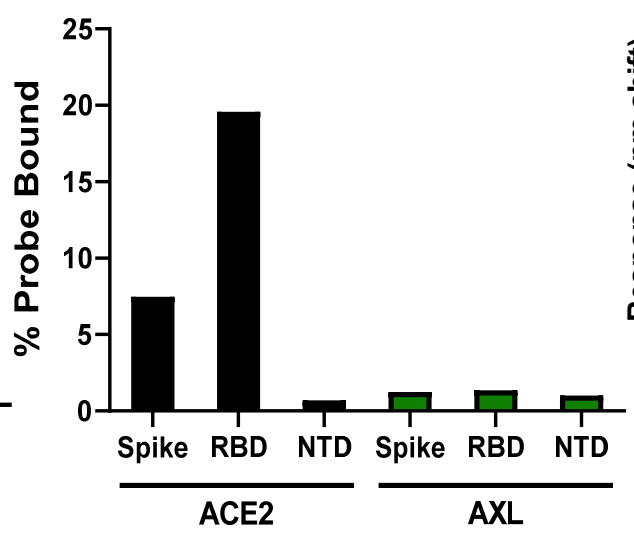

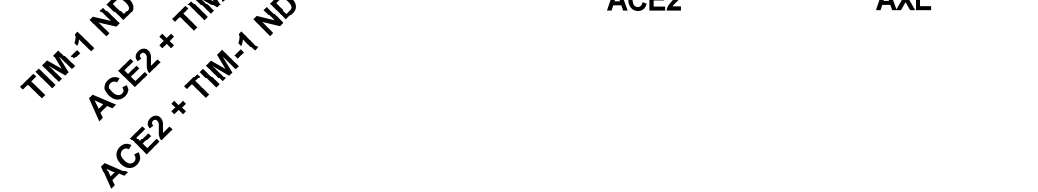


Figure 2: PS receptors interact with SARS-CoV-2 by binding to PS. A-B) PS liposomes interfere with rVSV-luciferase/Spike infection. HEK 293T cells transfected with TIM-1 plasmid and 50 ng of ACE2 plasmid (A) or AXL plasmid and 50 ng of ACE2 plasmid (B) were infected with rVSVluciferase/Spike in the presence of increasing concentrations of PS or PC liposomes and assessed for luciferase activity at 24 hours following infection. C) HEK 293T cells were transfected with WT or PS binding pocket mutant TIM-1 plasmids with or without 50 ng of ACE2 expressing plasmid and infected 48 hours later with rVSV-luciferase/Spike pseudovirions. Luminescence fold change were compared to mock transfected lysates that were set to a value of 1 . D) AXL is unable to directly interact with purified, soluble SARS-CoV-2 spike/Fc. HEK 293T cells transfected with AXL or ACE2 were incubated with soluble spike protein (S1/S2)-Fc, S1 RBD-Fc or S1 NTD-Fc and subsequently incubated with an Alexa 647 secondary. Spike protein binding was detected by flow cytometry. E) AXL does not bind to the NTD of SARS-CoV-2 spike. Biolayer interferometry association curves show that immobilized AXL-Fc fails to interact with purified NTD of spike.

Data are pooled from at least 3 independent experiments $(\mathbf{A}, \mathbf{B})$ or are representative of at least 3 experiments (C, D, E). Data represented as means \pm SEM. Multiple t-test (A, B), One-way ANOVA with multiple comparisons $(\mathbf{C})$; asterisks represent $\mathrm{p}<0.05$. 
Fig. 3. The route of SARS-CoV-2 entry is altered by TMPRSS2 expression

A
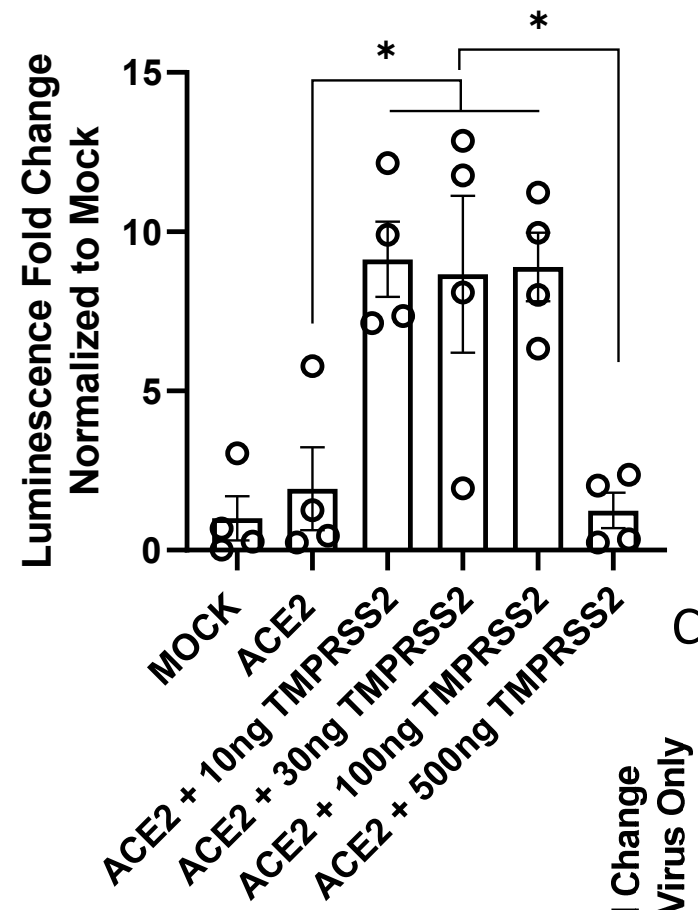

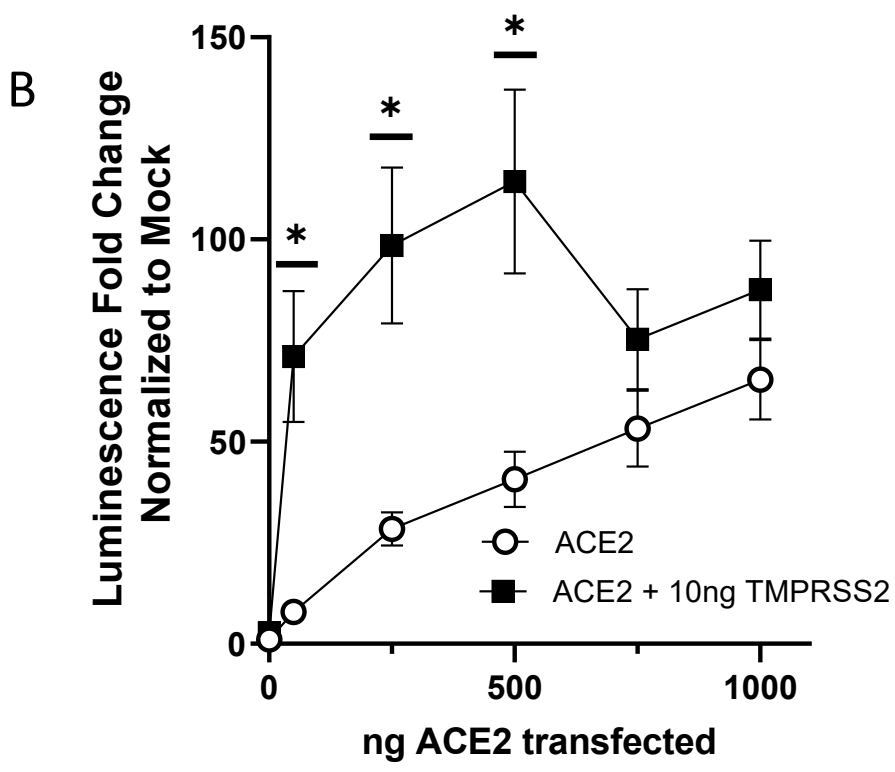

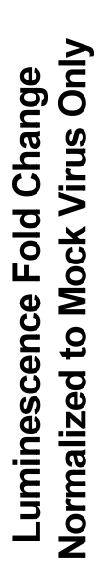

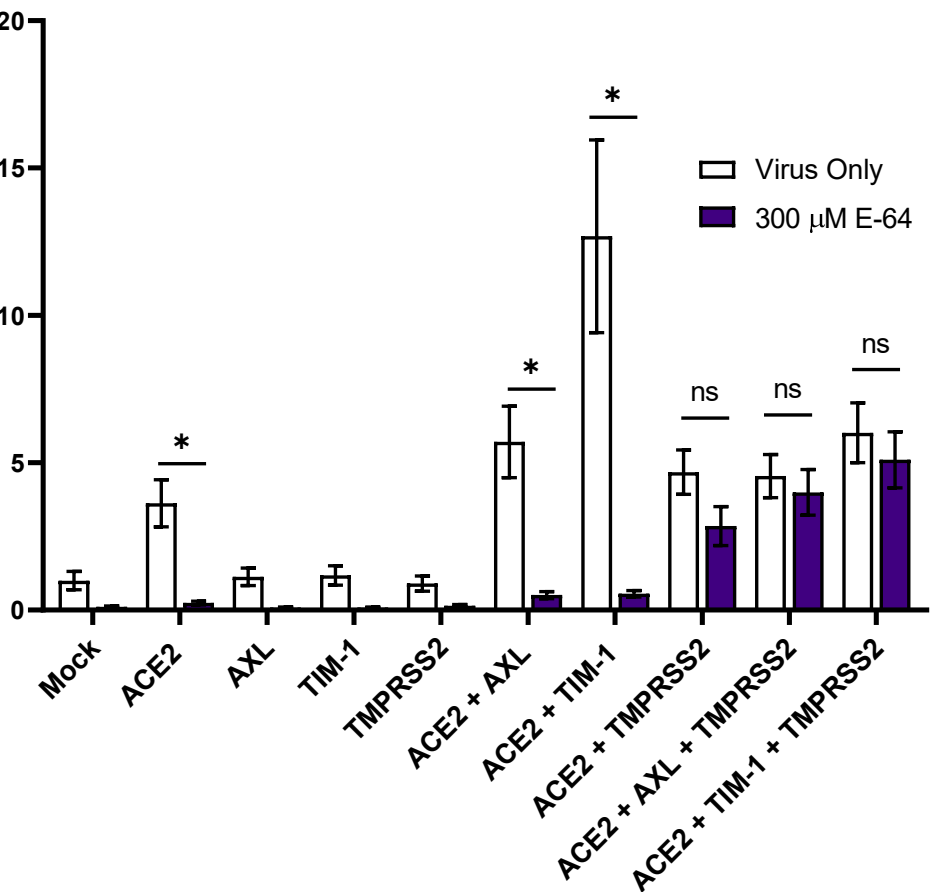


Figure 3: The route of SARS-CoV-2 entry is altered by TMPRSS2 expression. A) HEK 293T cells were transfected with ACE2 and TMPRSS2 as noted and infected at $48 \mathrm{~h}$ with VSV-luciferase/Spike. At 24 hpi, luminescence activity was determined. Findings are shown relative to empty vector (Mock) transfected cells. Panel depicts one representative experiment. Students t-tests. B) TMPRSS2 expression enhances rVSV-luciferase/Spike entry at low levels of ACE2 expression. HEK 293T cells were transfected as indicated and pseudovirion entry assessed by measuring luminescence activity at $24 \mathrm{hpi}$. C) Transfected HEK 293T cells were transfected and infected with VSV-luciferase/Spike at $48 \mathrm{~h}$ in the presence or absence of E-64 (300 $\mu \mathrm{M})$. Luciferase activity was determined $24 \mathrm{hpi}$.

Data are pooled from at least 3 independent experiments $(\mathbf{B}, \mathbf{C})$ or are representative of at least 3 experiments (A). Data represented as means \pm SEM. Student's T-tests (A) Multiple t-tests (B), Two-way ANOVA with row-wise multiple comparisons $(\mathbf{C})$; asterisks represent $p<0.05$. 
Figure 4: TAM inhibition reduces SARS-CoV-2 entry, binding, and infection.

A

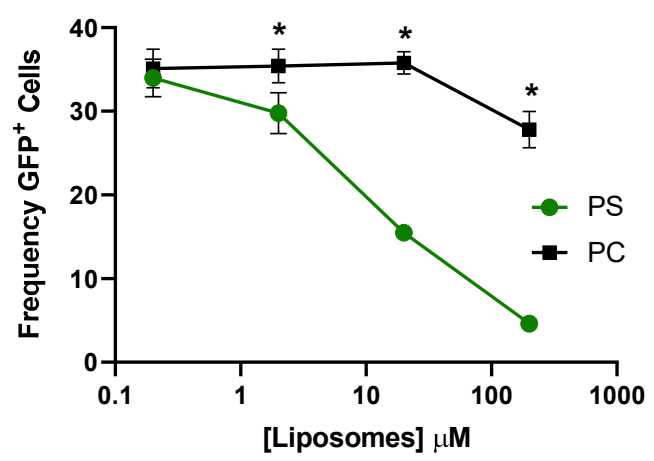

D

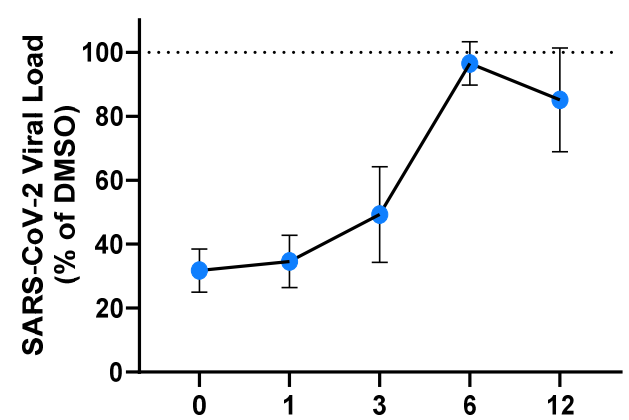

Hours Post-infection Bem. Added
B

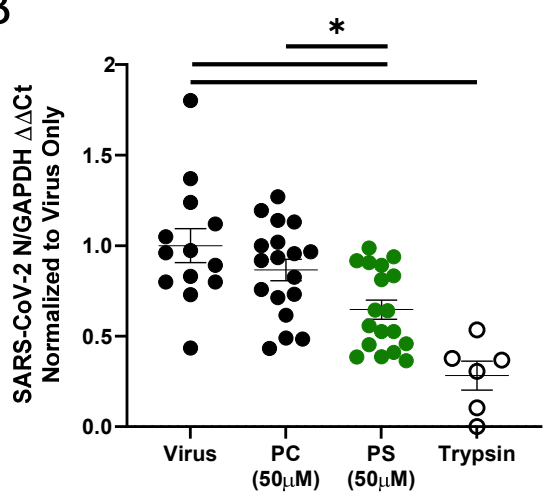

E

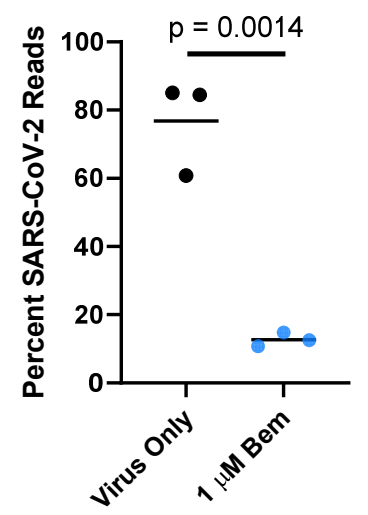

C

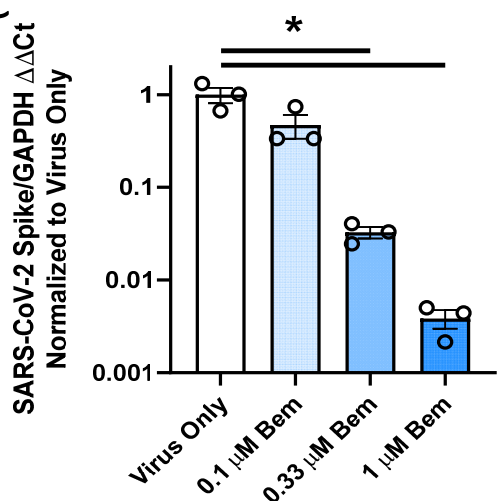

F

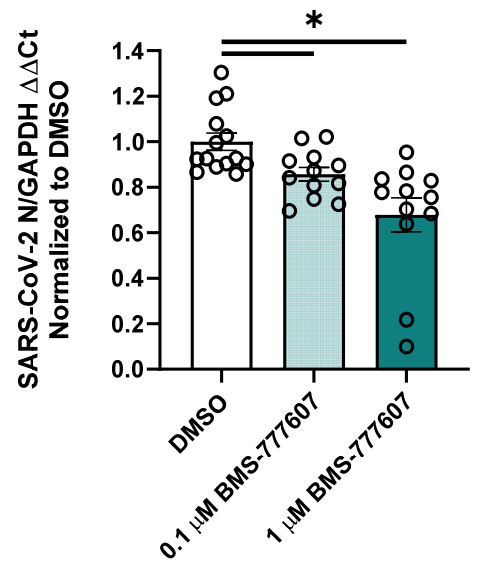

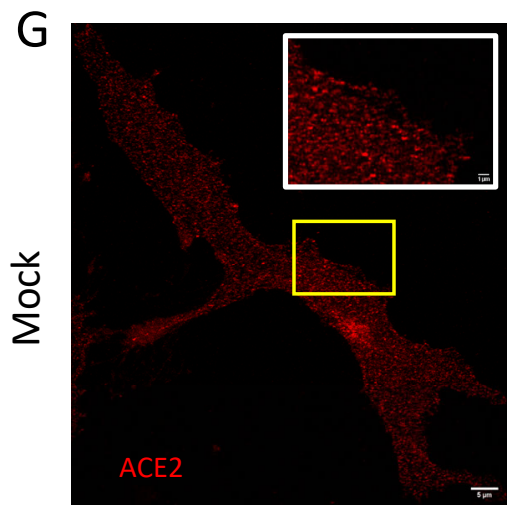

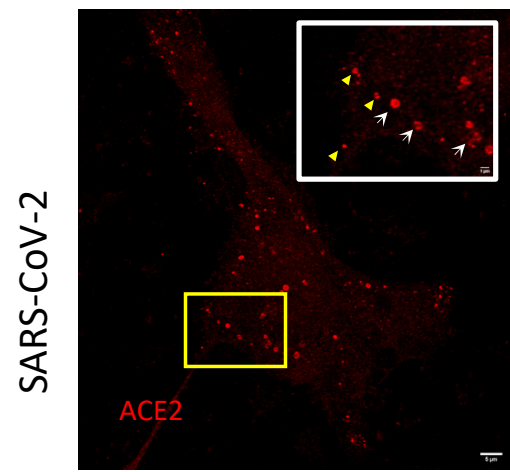

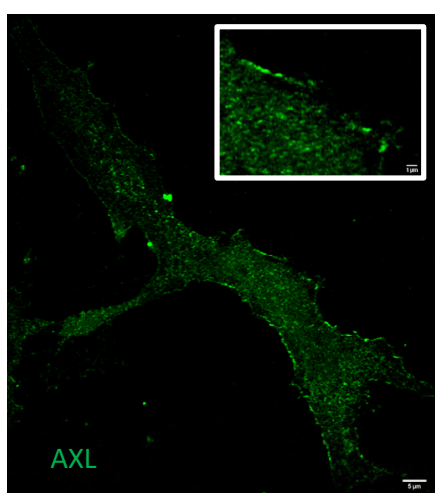

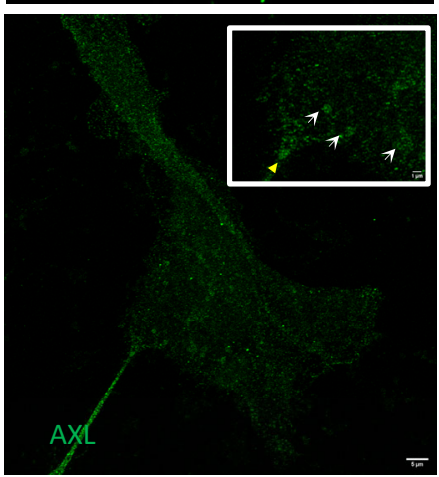

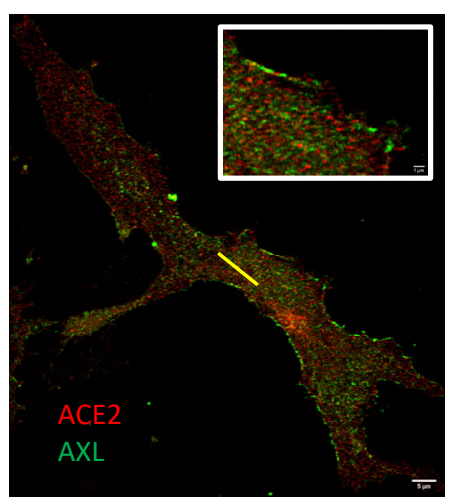
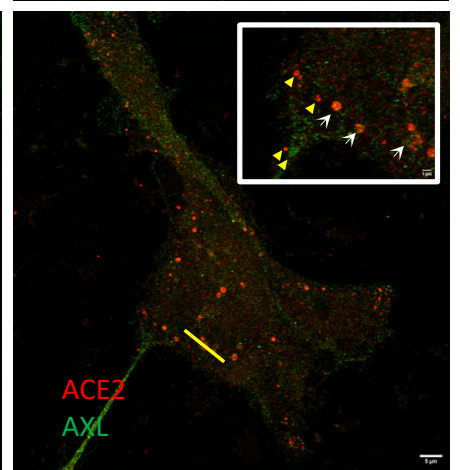

$\mathrm{H}$

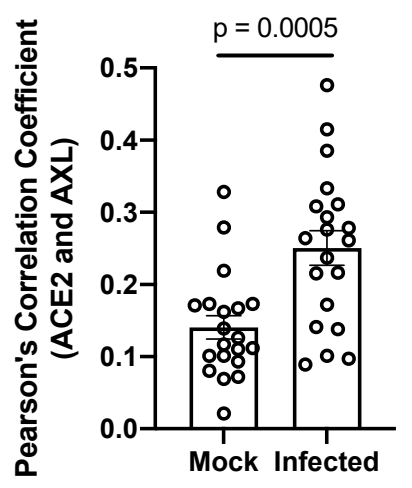


Figure 4: AXL has a prominent role in SARS-CoV-2 entry in Vero E6 cells. A) PS liposomes interfere with SARS-CoV-2 pseudovirion entry. Vero E6 cells were treated with PS or PC liposomes and incubated with VSV-GFP/Spike pseudovirions for 24 hours. Entry was detected by GFP fluorescence. B) PS liposomes disrupt SARS-CoV-2 binding. Vero E6 cells were incubated with SARS-CoV-2 (MOI $=5)$ at $10^{\circ} \mathrm{C}$ for 1 hour, washed extensively, and viral load assessed by RT-qPCR. C) AXL signaling inhibitor bemcentinib inhibits SARS-CoV-2 infection in Vero E6 cells. Cells were treated with bemcentinib and infected with SARS-CoV-2 $(\mathrm{MOI}=0.01)$. Viral loads were measured 24 hpi by RT-qPCR. D) Bemcentinib inhibition of SARS-CoV-2 infection is most efficacious at early time points during infection. Vero E6 cells were challenged with SARS-CoV-2 $(\mathrm{MOI}=0.01)$ and treated with either the vehicle control or $1 \mu \mathrm{M}$ bemcentinib at the indicated time. Viral loads were measured 24 hpi by RT-qPCR. E) Vero E6 cells were treated with $1 \mu \mathrm{M}$ bemcentinib, infected with SARS-CoV-2 (MOI $=0.01$ ) and mRNA harvested $18 \mathrm{hpi}$. mRNA was deep sequenced on an Illumina platform, and viral loads were calculated by alignment to the SARS-CoV-2 genome. F) Broad spectrum TAM inhibitor BMS777607 inhibits SARS-CoV-2 infection in Vero E6 cells. Cells were treated with inhibitor at indicated concentrations for 1 hour, challenged ( $\mathrm{MOI}=0.01)$, and viral loads measured $24 \mathrm{hpi}$ by RT-qPCR G) STED micrographs showing staining for ACE2 (red) and AXL (green) and merged in Vero E6 cells. Insets are enlarged images from regions highlighted by yellow rectangles. White arrows indicate shared vesicular structures between the two channels. Yellow arrowheads indicate objects that are only seen in one channel. Plot profiles are shown in S4F, representing signal intensity along the yellow lines in the merged panels. $\mathbf{H}$ ) Pearson's correlation coefficients of ACE 2 and AXL were calculated for $n=20$ mock and infected cells (ROI determined by cell borders).

Data are pooled from at least 3 independent experiments $(\mathbf{B}, \mathbf{D}, \mathbf{F})$ or are representative of at least 3 experiments $(\mathbf{A}, \mathbf{C}, \mathbf{G}, \mathbf{H})$. Data are represented as means \pm SEM. Multiple t-tests $(\mathbf{A})$ student's t-test $(\mathbf{B}, \mathbf{C}, \mathbf{F}, \mathbf{H})$; asterisks represent $\mathrm{p}<0.05$. 
Figure 5: AXL inhibition reduces SARS-CoV-2 infection in human lung cells lines

A

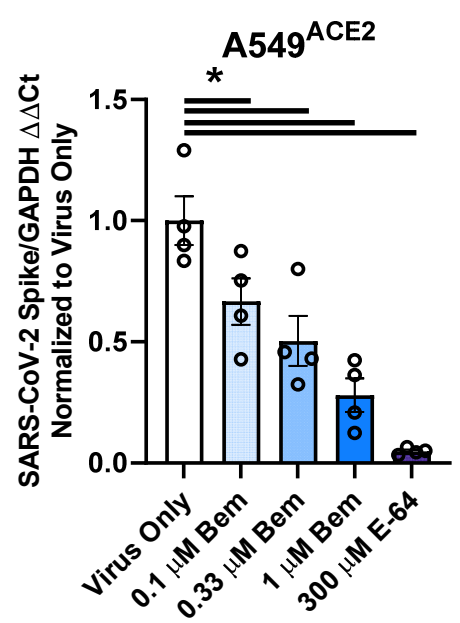

D

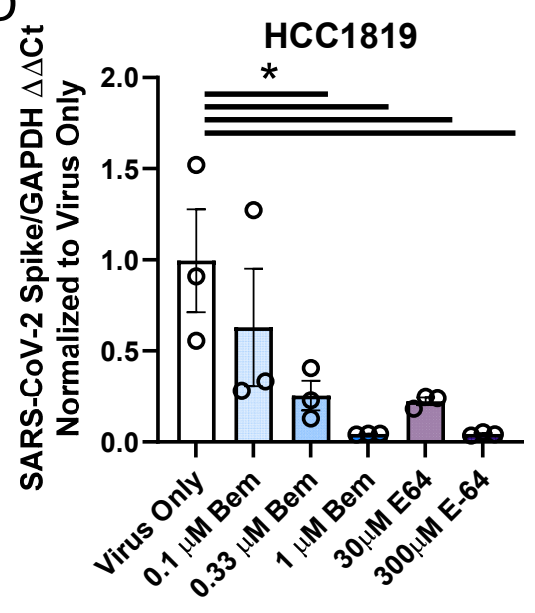

G ثّ

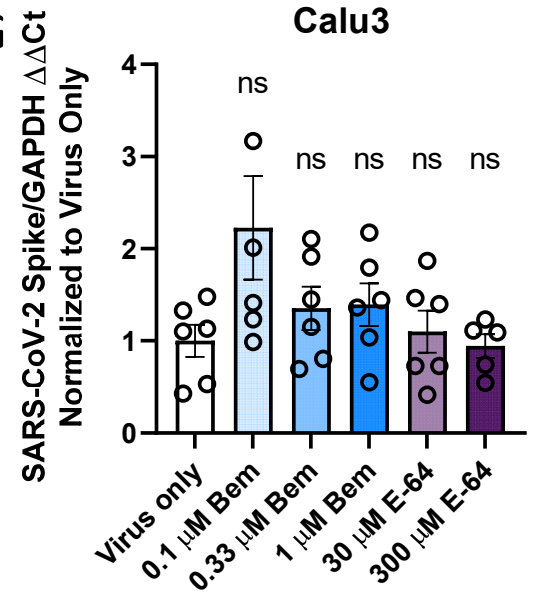

B

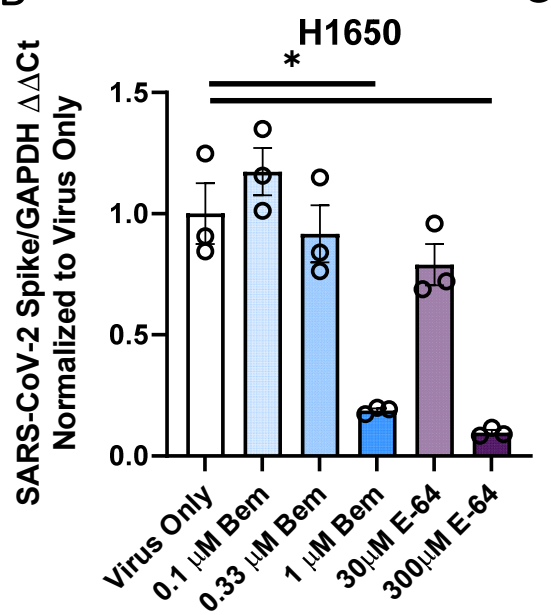

$\mathrm{E}$

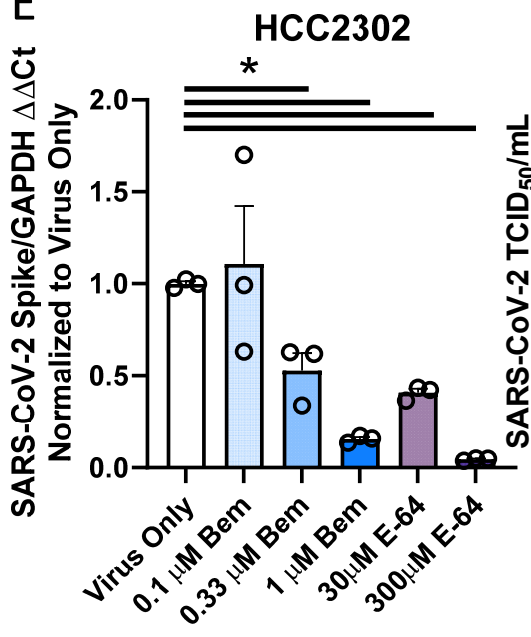

A549ACE2

C

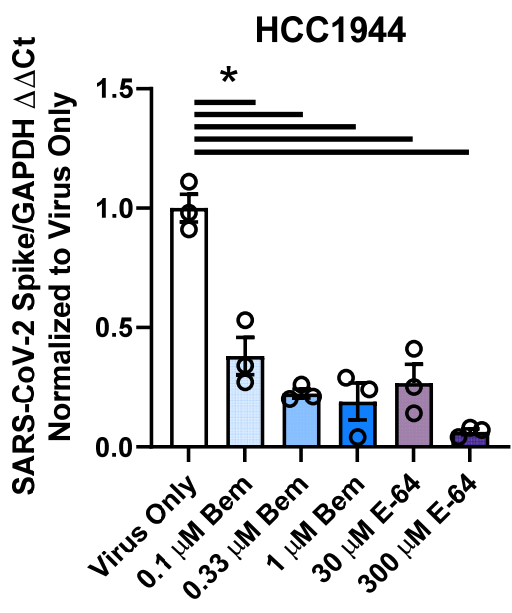

HCC2302
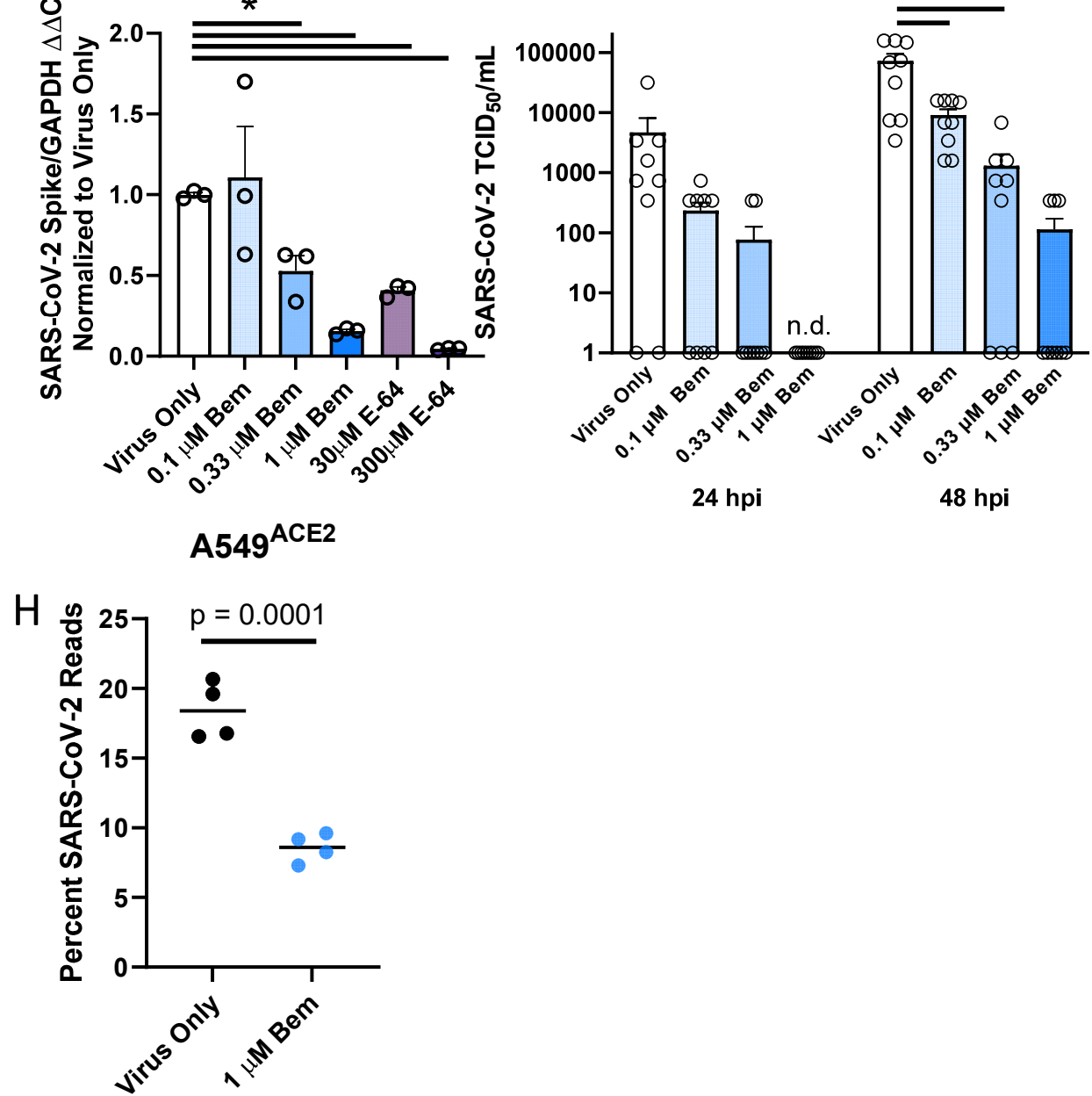
Figure 5: AXL inhibition reduces SARS-CoV-2 infection in human lung cells. A-F) SARS-CoV2 infection is reduced by AXL inhibition in multiple human lung cell lines. In order: A549ACE2, H1650, HCC1944, H1819, HCC2302, Calu3 were treated with the indicated inhibitors for 1 hour and challenged with SARS-CoV-2 $(\mathrm{MOI}=0.5)$ for 24 hours. Viral load was assessed by RT-qPCR. G) HCC2302 cells were treated with bemcentinib at the indicated concentrations for 1 hour and infected with SARS-CoV-2 (MOI =0.5). Input virus was removed $6 \mathrm{hpi}$ and supernatant was collected at 24 and $48 \mathrm{hpi}$ and titered by $\mathrm{TCID}_{50}$ assays on Vero E6TMPRSS2 cells. TCID $50 / \mathrm{mL}$ was calculated by the Spearmann-Karber method. H) A549ACE2 were treated with bemcentinib as indicated, infected with SARS-CoV-2 $(\mathrm{MOI}=0.5)$ and mRNA harvested $24 \mathrm{hpi}$. mRNA was sequenced, and viral loads calculated by alignment to the SARS-CoV-2 genome.

Data are pooled from at least 3 independent experiments (F) or are representative of at least 3 experiments (A, B, C, D, E, G). Data represented as means \pm SEM. Student's t-test; asterisks represent $p<0.05$. 
Figure 6: AXL knockout dramatically reduces viral loads and ablates inhibition by bemcentinib.

A
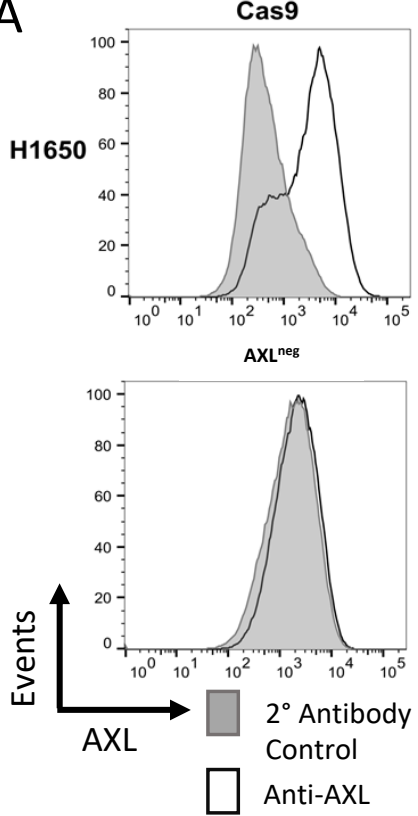

B
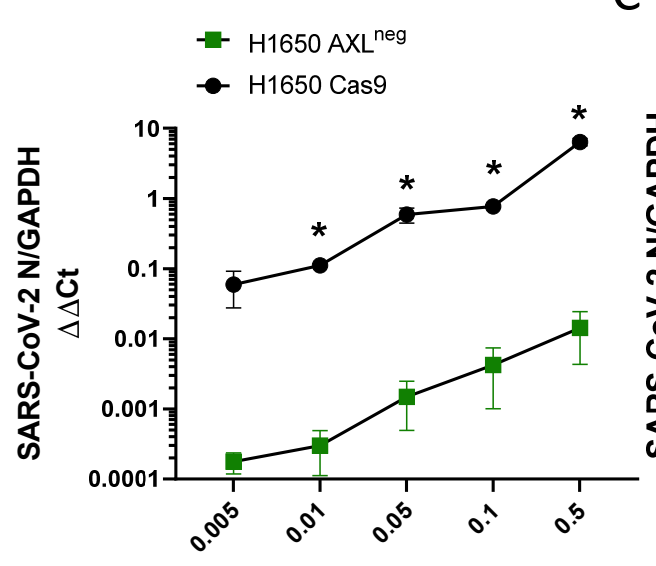

SARS-CoV-2 MOI
C

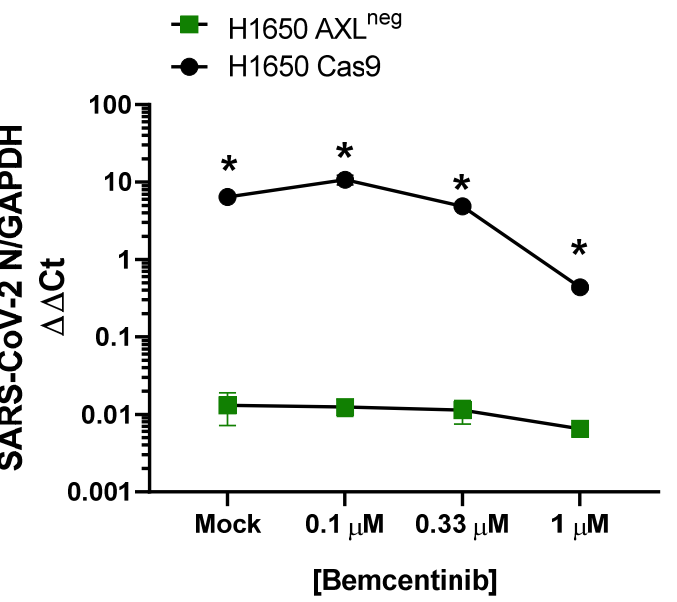


Figure 6: AXL knockout reduces viral loads and ablates inhibition by bemcentinib. A) H1650 AXL knockout cells were generated by lentiviral transduction of Cas 9 and gRNA targeting $A X L$, followed by selection, enrichment, and biological cloning. Shown are flow cytometry histograms depicting AXL surface staining (black) and secondary only background (grey), demonstrating loss of AXL expression. B) H1650 AXL neg and H1650 Cas9 (parental) lines were challenged with SARS-CoV-2 at indicated MOls. At $24 \mathrm{hpi}$, viral loads were assessed by RT-qPCR. C) H1650 parental and AXL neg lines were treated with indicated concentration of bemcentinib for 1 hour and subsequently challenged with SARS-CoV-2 $(\mathrm{MOI}=0.5)$ and viral loads determine by RT-qPCR $24 \mathrm{hpi}$.

Data are pooled from at least 3 independent experiments $(\mathbf{B}, \mathbf{C})$ or are representative of at least 3 experiments $(\mathbf{A})$. Data represented as means \pm SEM. Multiple t-tests; asterisks represent $p<0.05$. 